\title{
Modeling of the Land Mobile Satellite Channel considering the Terminal's Driving Direction
}

\author{
Marie Rieche, ${ }^{1}$ Alexander Ihlow, ${ }^{1}$ Daniel Arndt, ${ }^{1}$ \\ Fernando Pérez-Fontán, ${ }^{2}$ and Giovanni Del Galdo ${ }^{1}$ \\ ${ }^{1}$ Institute for Information Technology, Technische Universität Ilmenau, Helmholtzplatz 2, 98693 Ilmenau, Germany \\ ${ }^{2}$ Telecommunication Engineering School, University of Vigo, 36310 Vigo, Spain \\ Correspondence should be addressed to Marie Rieche; marie.rieche@tu-ilmenau.de
}

Received 9 May 2014; Revised 4 August 2014; Accepted 5 August 2014

Academic Editor: Jose F. Paris

Copyright (C) 2015 Marie Rieche et al. This is an open access article distributed under the Creative Commons Attribution License, which permits unrestricted use, distribution, and reproduction in any medium, provided the original work is properly cited.

\begin{abstract}
A precise characterization of the Land Mobile Satellite (LMS) channel, that is, the channel between a satellite and a mobile terminal, is of crucial importance while designing a satellite-based communication system. State-of-the-art statistical LMS channel models offer the advantage of requiring only a few input parameters, which include the environment type and the elevation angle of the satellite. However, the azimuth angle relative to the driving direction of the mobile terminal is usually ignored, as its proper modeling requires either an extensive measurement campaign or a significant effort from the user, as a precise geometrical description of the scenario is required. In this contribution we show that the impact of the driving direction on the channel statistics is not negligible and requires to be modeled explicitly. Moreover, we propose a statistical LMS channel model whose parameters are obtained via an image-based state estimation method. The image-based method is verified by a comparison with measured radio frequency signal levels. The proposed method allows obtaining a complete statistical description of the channel for arbitrary elevation and azimuth angles.
\end{abstract}

\section{Introduction}

Satellite communication services need to ensure a certain quality of the received signal. For stationary applications, this is a relatively undemanding task, as it is normally possible to provide a nearly ideal Line-of-Sight (LoS) connection. However, this is not the case for moving terminals. In order to design appropriate systems for this specific application, a profound knowledge of the propagation channel, the so-called land mobile satellite (LMS) channel, becomes necessary.

The LMS channel is mainly impaired by the environment in the direct vicinity of the moving terminal. In fact, the LoS component may be completely obstructed by solid objects (e.g., a building) or may be more or less shadowed by vegetation depending on the density of the foliage and branches. Moreover, diffraction, reflection, and scattering of the transmitted signal generate multipath components.

Looking at the received signal level over traveled distance, as in Figure 1, it is possible to distinguish different effects: the LoS component (which might be blocked or not) gives rise to "very slow fading", while in a partially obstructed condition, it gives rise to "slow fading", for example, due to the vegetation. Furthermore, the multipath propagation introduces "fast fading", which is superimposed to slow and very slow fading. Besides the impact on the signal level described above, the channel also causes temporal dispersion (induced by the different lengths of the propagation paths) and the Doppler shifts, as terminal, satellite, and possibly some objects in the environment are moving.

The LMS channel can be modeled with different approaches, where statistical channel models are frequently used and are able to generate time-series of the received fading signal [1-3]. Their main advantage, compared to other approaches, is that they do not require many input parameters and offer the possibility to generate a large quantity of different channels. These models usually feature two different input parameters: the environment type (e.g., urban, suburban) and the elevation angle of the satellite. 


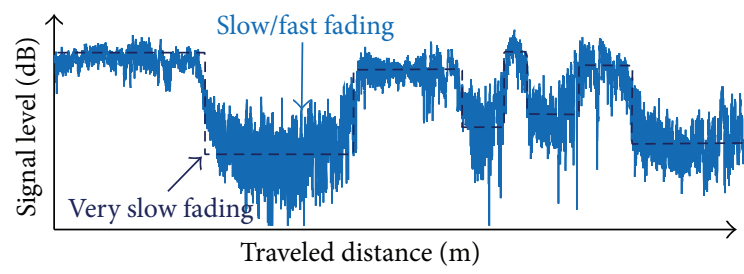

FIGURE 1: The received satellite signal is characterized by a superposition of very slow, slow, and fast fading effects.

Normally, they do not consider the driving direction of the mobile terminal, that is, the azimuth angle of the satellite. Ignoring the driving direction in the model results in a timeseries of the signal fading, which is stationary with respect to the driving direction. This is intuitively an oversimplification for urban scenarios, where a satellite oriented in driving direction leads to a higher signal quality compared to a satellite perpendicular to the driving direction. This is exemplified in Figure 2.

Traditionally, the internal model parameters are derived from statistical distributions, whose parametrization is based on Radio Frequency (RF) measurements. The measurement data utilized by the statistical LMS models, available in the literature, is gathered either from one or more satellites at specific azimuth positions or from a helicopter emulating the satellite. If measurements are carried out for several satellites at different azimuth positions, the average over the available satellite positions in azimuth is calculated. Consequently, the extracted model parameters reflect the flight/satellite conditions and the angle between the radio path and the mobile but do not explicitly consider it. To develop an LMS model which explicitly considers the driving direction, it is necessary to collect enough RF measurements to carry out a statistical analysis in dependence of the relative azimuth angle. For instance, by dividing the full circle $\left(360^{\circ}\right)$ into intervals of $10^{\circ}$ each, one needs 36 times the measurement data to achieve an equivalent statistical significance.

In this contribution we extend the statistical LMS model for S-Band proposed by Arndt et al. [5]. We consider the driving direction with a resolution of $1^{\circ}$ over the whole azimuthal range of $360^{\circ}$. To solve the challenging task of gathering the necessary large amount of RF measurement data, we follow an alternative image-based approach [23], which allows us to extract the very slow fading of the received signal for arbitrary positions of the satellite, even for those which are not yet in orbit. The method enables the derivation of accurate parameters for different elevations and especially different driving directions with an angular resolution of $1^{\circ}$. Based on the high azimuthal resolution we found that the left-right symmetry assumed in [5] is an oversimplification, because the vehicle travels on one side of the street. We show the significant impact of considering the azimuthal satellite position relative to the driving direction, in contrast to neglecting the azimuthal position, on the quality of the received signal in terms of first order statistics.

The remainder of this contribution is organized as follows: Section 2 gives an overview of state-of-the-art LMS channel models and closer analyzes those, feasible to reflect the effects of the driving direction. Section 3 illustrates the image-based state estimation method, which allows the extraction of the very slow fading, Furthermore, the accuracy of this method is shown by comparing with simultaneously recorded RF signal levels. Section 4 introduces the main components of the LMS model. Section 5 presents the impact of the driving direction on the statistics of the satellite signal. Finally, Section 6 summarizes the main results of the contribution.

\section{Modeling Approaches of the Land Mobile Satellite Channel}

This section concisely reviews different principles of LMS channel modeling and explains the most common LMS channel models.

It is convenient to distinguish between four basic principles [24].

(i) Empirical channel models describe the signal attenuation by fitting mathematical expressions to measurements, without including any physical interpretation. An overview of different empirical LMS channel models can be found in [25].

(ii) Deterministic channel models describe the propagation effects for specific environments, while approximating Maxwell's equations. Most of them are based on assumptions of a propagating electromagnetic wave and are so-called ray-based. Ray-tracing approaches applied to the LMS channel are described in [26].

(iii) Statistical channel models describe the channel in terms of statistical distributions, which may or may not have physical meaning. The parameters of these models are extracted from a representative number of measurements.

(iv) Hybrid channel models are a combination of the approaches mentioned above. In the literature, a large variety of these models can be found, making them difficult to categorize. When deterministic and physical channel models are combined, they are so-called physical-statistical models. A synthetic propagation environment is generated by considering deterministic/physical techniques, whereas statistical distributions are utilized for a description of the environment or the signal propagation.

Furthermore, we can identify more categories of available LMS channel models depending on other features.

(i) One feature for categorizing LMS channel models is the number of reception states supported. The received satellite signal can no longer be assumed stationary for a longer time period. Different reception states are introduced to represent the block-wise stationarity of the received signal, which is caused by the slow varying environmental conditions (like LoS or NLoS due to, e.g., buildings, trees), while the terminal moves. 


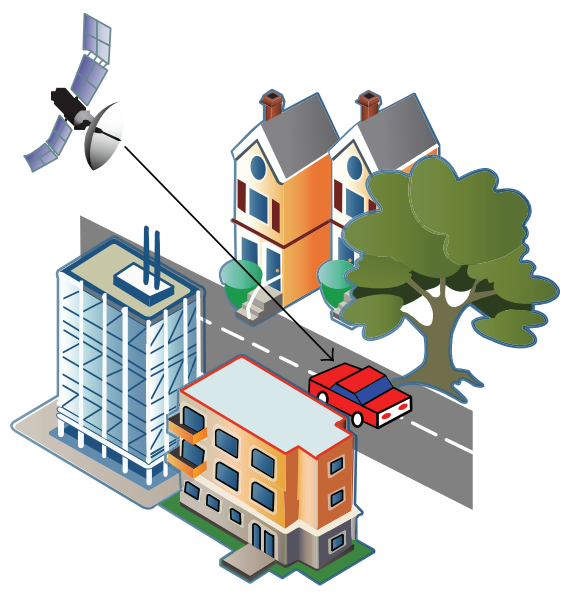

(a) Line-of-sight (LoS)

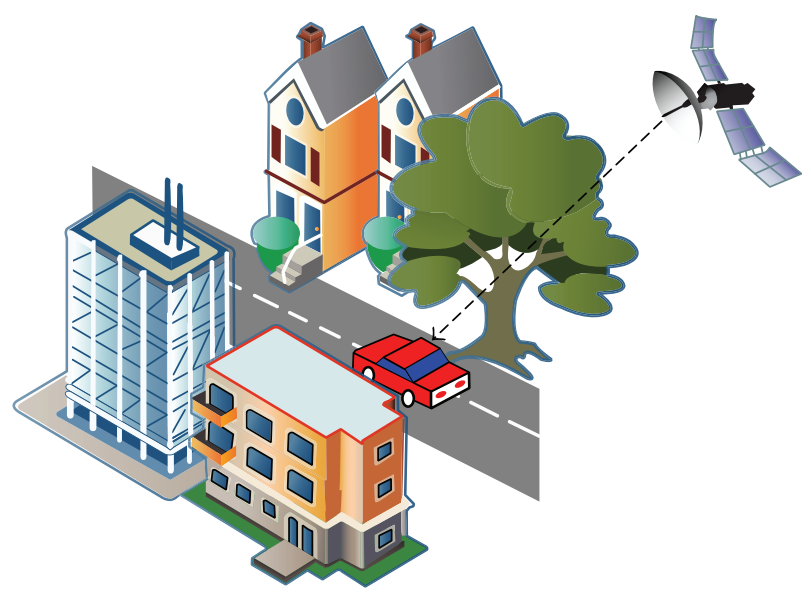

(b) Non-line-of-sight (NLoS)

Figure 2: The mobile user can be situated in different receiving states, which depend on the surrounding objects and the azimuthal angle relative to the driving direction.

Single-state LMS channel models are those which only consider the slow and fast fading of the received satellite signal. Over a short time period these two components (slow and fast fading) are modeled by a stationary stochastic processes, for example, Loo [27-29]. Multistate LMS channel models differentiate two states ("good" and "bad") [30], three states ("lineof-sight," "shadowed," and "blocked") [31] or higher (variable) number of states [32]. The sequence of reception states can either be generated by a Markov process or by physical/geometrical considerations of the environment (see Section 2.2).

(ii) Besides the number of states we can also distinguish between LMS channel models for different number of satellites. In the literature, several single-satellite models can be found $[1,2,5,30]$. Available multisatellite models usually consider dual-satellite systems [3, $4,6]$. Lutz [3] already emphasizes the impact of the correlation between two satellite links, as it provides information about the visibility of satellites and the diversity gain. Propagation models which consider more than two satellites (e.g., $[7,33]$ ) are rather rare in the literature, due to the more complex description of the satellites correlations.

In the following we deal more in detail with the purely statistical and the physical-statistical approaches, as they provide the possibility to include the driving direction and offer less complexity, compared to purely deterministic models.

2.1. Statistical Land Mobile Satellite Channel Models. Stateof-the-art purely statistical models [2], which produce timeseries of the envelope of the received signal, derive their parameters from RF measurements. They implicitly include the driving direction without evaluating it adequately. If the driving direction was considered explicitly in the channel modeling, the resulting statistics were more realistic for specific situations, as shown in Section 5. Therefore, it would be necessary to collect a sufficient amount of RF data to carry out a statistical analysis in dependence of the relative azimuth angle. In [1] the measurement data was conducted for relative azimuth angles of approximately $0^{\circ}, 45^{\circ}$, and $90^{\circ}$. Arndt et al. [5] utilize measurement data for different environments, elevation angles, and driving directions. The model consists of a state sequence generator implemented as semi-Markov chains and L Loo-generator simulating slow and fast fading effects within each state. The model parameters are derived from extensive measurements of RF signal levels in S-Band for different environments, elevation angles, and driving directions. The intervals of driving directions with respect to the satellite are $0^{\circ} \cdots 10^{\circ}, 10^{\circ} \cdots 30^{\circ}, 30^{\circ} \cdots 60^{\circ}$, and $60^{\circ} \cdots 90^{\circ}$. This model assumes a symmetry of $90^{\circ}$ for the different driving directions and clusters the data accordingly, which is an oversimplification, as we show in Section 5.

\subsection{Physical-Statistical Land Mobile Satellite Channel Models.} There are several hybrid LMS channel models available, which consider the driving direction by different geometrybased approaches. The "virtual city" approach represents a special case for the urban environment. Building heights, street widths building block lengths, and so forth are modeled following a statistical distribution. The output of these models are multiple state sequences, which allow to calculate the correlation factors between different satellite links of azimuth and elevation. One of the first physical-statistical LMS models is introduced by Tzaras et al. [8]. The basis is a physical approach based on synthetic environments, where the heights of obstacles follow certain distributions (or are assumed to be constant). The mobile terminal can also be shifted away from the middle of the street. The result is presented in terms of an analytic expression based on diffraction theory, depending only on the azimuth angle separation.

Oestges et al. [9] develop a prediction of the statistical fade distribution based on a conditional Rice distribution. The parameters of the Rician distribution vary with the 
physical parameters and depend on the satellite position (elevation and azimuth) and on the environment (building height and street width). The relationship between the Rice parameters and the physical parameters are validated by raytracing, based on the Uniform Theory of Diffraction (UTD). Building height and street width are assumed to be lognormally distributed, based on measurements in London and Guildford. The results are shown for an azimuth angle of $90^{\circ}$ relative to the street axis and the vehicle was positioned in the middle of the street. Based on this model, the shadowing correlation coefficient for a dual-satellite system as a function of the azimuth separation is presented in [10]. The correlation model is based on the Rician dominant component of the physical-statistical model explained in [9]. Here, the vehicle is positioned at one side of the road, which shows the effect of the right-hand side and left-hand side driving, respectively. In [11], again the same geometry-based approach is used to model time-series, combined with a series of roadside buildings according to measured distributions of geometrical parameters such as building height, street width, and height of the nearest building. This model considers the position of the car to be in the middle of the street.

There are several models based on a street mask concept, mask angle (MKA) or mask function (MKF), respectively [12-16]. These graphical representations of the azimuth and elevation angle indicate the visibility of satellites from the mobile terminal's point of view for different urban scenarios (e.g., street canyons, street crossings). The input parameters are building height, terminal height, and street width, whereby the user is assumed to be in the middle of the street, therefore the correlation between two satellite links is symmetric. To generate the building heights and street widths statistical distributions are used.

A hybrid statistic-deterministic model [17] is based on a Markov chain, considering elevation and street orientation angles (assuming symmetry of $90^{\circ}$ ). The visibility of the satellite is also described by the MKA, when the terminal is located in the middle of the street. The resulting "on/off" time-series is enhanced by multipath and diffraction effects based on a simple ray-tracing model (statistical distributions for building height and width are used).

A methodology, which uses also fisheye images to derive the state information (clear, shadowed, and blocked) and adding different distributions (i.e., Loo, Rayleigh, and Rice) to simulate the fast fading effects, can be found in [1921, 34]. Meenan et al. [18] also use fisheye images and apply the approach to the correlated four-state Lutz model [3]. However, none of these models explicitly takes into account the dependency on azimuth angle relative to the street orientation.

Table 1 gives an overview of the statistical and physicalstatistical models, which are concisely reviewed in this section. These models are frequently used in the literature and differ in their assumptions, constraints, and outputs. Therefore, the models are compared with respect to their modeling technique, the number of satellites, if they consider the driving direction of the terminal, as well as the correlation coefficient (corr). Further evaluation criteria are the cumulative distribution function (CDF) and the bad state probability $p_{b}$ of a satellite.

In summary, state-of-the-art LMS channel models are either uncapable of describing the influence of the azimuth angle of the satellite due to the lack of data, or they necessitate significant effort from the user, as a precise description of the geometry of the environment is required.

\section{State Parameter Extraction}

As mentioned in the introduction, to carry out a statistical analysis for different driving directions it is necessary to collect an unfeasible amount of measurement data. To solve this problem, we rely on the image-based method described in [35], which is reviewed in the next Section 3.1. This method is capable of estimating the very slow fading conditions of the LMS channel for an arbitrary position of the satellite using hemispheric images of the environment surrounding the mobile terminal. The very slow fading is modeled with a sequence of the reception states $\mathrm{good}$ and bad, corresponding to line-of-sight (LoS) and nonline-of-sight (NLoS), respectively [2]. Section 3.2 presents the measurement campaign realized to collect the necessary images for the model proposed here. Finally, Section 3.3 addresses the validation of the image-based method by comparing its estimates with actual RF measurements.

3.1. Image-Based State Estimation Method. In the following, we review the image-based method from which we derive the LMS channel reception states [35]. Hemispheric images of the environment are obtained from a fisheye camera pointing towards the sky. Such an image of the upper hemisphere is depicted in Figure 3(a). The image is classified in the region $s k y$, where the satellite is visible and the region obstruction, where it is shadowed. The result is shown in Figure 3(b) as an overlay of the binary classification result and the original image. Finally, the classified fisheye image is converted into a rectangular image in landscape panoramic form yielding a resolution of one degree in elevation and azimuth (see Figure 3(c)). Knowing the time, location, and heading information of the vehicle we can determine the position of arbitrary satellites within the image given their azimuth and elevation with respect to a known coordinate system. From the position of the satellite within the panoramic image we can directly extract the reception state, where sky represents the good state and obstruction represents the bad state, respectively. Based on the extracted state sequence we can determine different state parameters, representing an essential part of the LMS channel model parameters, as explained in detail in Section 4.

3.2. Measurement Data Acquisition. To validate the proposed method we consider extensive measurements conducted in the context of the project Mobile satellite channeL with Angle DiversitY (MiLADY) [36]. During this campaign the received power levels of two geostationary (GEO) satellites of XM Satellite Radio and two of three highly elliptical orbit (HEO) 
TABLE 1: Statistical and physical-statistical LMS channel models are compared with respect to the modeling approach, the number of satellites, considering the driving direction of the terminal, considering the correlation coefficient (Corr) and regarding the evaluation criteria cumulative distribution function $(\mathrm{CDF})$ and the bad state probability of a satellite $p_{b}$.

\begin{tabular}{|c|c|c|c|c|c|c|c|}
\hline Modeling approach & Proposed by & Year & Number of satellites & Driving direction considered & $p_{b}$ & Corr & $\mathrm{CDF}$ \\
\hline \multirow{7}{*}{ Statistical } & Pérez-Fontán et al. [1] & 2001 & Single & & & & $\mathrm{x}$ \\
\hline & Prieto-Cerdeira et al. [2] & 2010 & Single & & & & $\mathrm{x}$ \\
\hline & Lutz $[3]$ & 1996 & Dual & & $\mathrm{x}$ & $\mathrm{x}$ & \\
\hline & Arndt et al. [4] & 2012 & Dual & $\mathrm{x}$ & & & \\
\hline & Arndt et al. [5] & 2012 & Single & $\mathrm{x}$ & $\mathrm{x}$ & & $\mathrm{x}$ \\
\hline & Arndt et al. [6] & 2012 & Dual & $\mathrm{x}$ & $\mathrm{x}$ & $\mathrm{x}$ & \\
\hline & Arndt et al. [7] & 2012 & Multi & & & $\mathrm{x}$ & \\
\hline Physical-statistical & Tzaras et al. [8] & 1998 & Dual & & & $\mathrm{x}$ & \\
\hline \multirow{3}{*}{ Ray-tracing } & Oestges et al. [9] & 1999 & Single & & & & $\mathrm{x}$ \\
\hline & Oestges and Vanhoenacker-Janvier [10] & 2001 & Dual & & & $\mathrm{x}$ & \\
\hline & Oestges and Vanhoenacker-Janvier [11] & 2007 & Dual & & & $\mathrm{x}$ & $\mathrm{x}$ \\
\hline \multirow{5}{*}{ MKA } & Vázquez-Castro et al. [12] & 2001 & Dual & & & $\mathrm{x}$ & \\
\hline & Vázquez-Castro et al. [13] & 2002 & Dual & & & $\mathrm{x}$ & \\
\hline & Pérez-Fontán et al. [14] & 2002 & Multi & & & $\mathrm{x}$ & \\
\hline & Pérez-Fontán et al. [15] & 2002 & Multi & & $\mathrm{x}$ & & \\
\hline & Pérez-Fontán et al. [16] & 2003 & Multi & & $\mathrm{x}$ & & \\
\hline Virtual city & Pérez-Fontán et al. [17] & 2005 & Single & $\mathrm{x}$ & & & \\
\hline \multirow{3}{*}{ Fisheye } & Meenan et al. [18] & 1998 & Dual & & & $\mathrm{x}$ & \\
\hline & Akturan et al. [19-21] & 1997 & Single & & & & $\mathrm{x}$ \\
\hline & Rieche et al. [22] & 2014 & Single & $\mathrm{x}$ & & & $\mathrm{x}$ \\
\hline
\end{tabular}

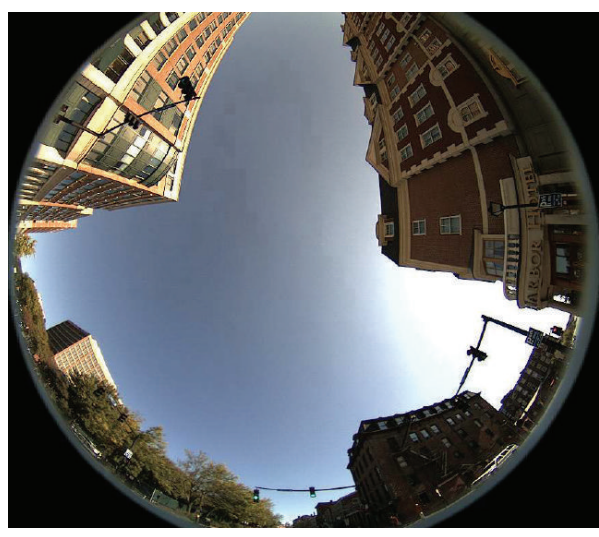

(a) Exemplary hemispheric image

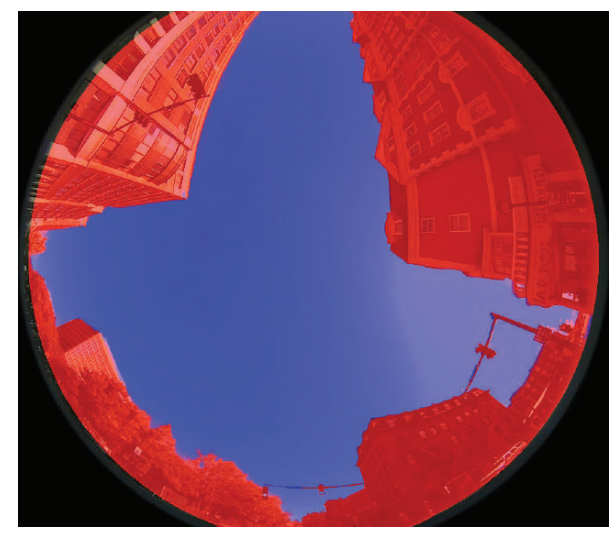

(b) Overlay of the original and the binary classification into sky (blue) and obstruction (red)

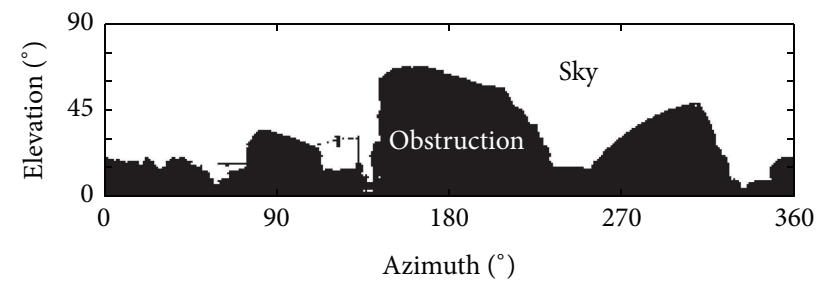

(c) The converted panoramic binary images are of size $90 \times 360$ pixels, where the resolution is one degree in elevation and in azimuth. White represents the reception state good and black bad, respectively

FIGURE 3: The images show the original hemispheric image, the result of the image classification, and the converted panoramic binary image, where the reception state good and bad is categorized. 
TABLE 2: Semi-Markov parameters (good and bad state) for urban environment for variable driving directions and elevations.

\begin{tabular}{|c|c|c|c|c|c|c|c|c|}
\hline Elevation & Front & Front-right & Right & Back-right & Back & Back-left & Left & Front-left \\
\hline \multicolumn{9}{|c|}{ Log-normal mean $\mu_{\text {dur,bad }}$} \\
\hline 85 & 1.28 & 1.28 & 1.29 & 1.33 & 1.29 & 1.25 & 1.24 & 1.30 \\
\hline 80 & 1.28 & 1.29 & 1.32 & 1.29 & 1.25 & 1.15 & 1.11 & 1.18 \\
\hline 75 & 1.29 & 1.41 & 1.45 & 1.35 & 1.20 & 1.11 & 1.18 & 1.19 \\
\hline 70 & 1.30 & 1.45 & 1.53 & 1.39 & 1.20 & 1.13 & 1.25 & 1.17 \\
\hline 65 & 1.30 & 1.54 & 1.60 & 1.50 & 1.23 & 1.22 & 1.42 & 1.16 \\
\hline 60 & 1.32 & 1.60 & 1.66 & 1.60 & 1.27 & 1.42 & 1.61 & 1.29 \\
\hline 55 & 1.39 & 1.65 & 1.70 & 1.64 & 1.29 & 1.57 & 1.71 & 1.46 \\
\hline 50 & 1.43 & 1.71 & 1.81 & 1.70 & 1.35 & 1.68 & 1.76 & 1.58 \\
\hline 45 & 1.49 & 1.80 & 1.90 & 1.78 & 1.40 & 1.74 & 1.82 & 1.66 \\
\hline 40 & 1.55 & 1.85 & 1.90 & 1.85 & 1.50 & 1.82 & 1.85 & 1.75 \\
\hline 35 & 1.61 & 1.93 & 1.99 & 1.94 & 1.59 & 1.85 & 1.87 & 1.82 \\
\hline 30 & 1.67 & 1.99 & 2.06 & 2.07 & 1.68 & 1.87 & 1.83 & 1.84 \\
\hline 25 & 1.72 & 2.05 & 2.16 & 2.22 & 1.80 & 1.94 & 1.96 & 1.92 \\
\hline 20 & 1.83 & 2.11 & 2.22 & 2.35 & 1.87 & 2.10 & 2.09 & 2.05 \\
\hline 15 & 2.04 & 2.21 & 2.27 & 2.50 & 2.09 & 2.22 & 2.16 & 2.18 \\
\hline \multicolumn{9}{|c|}{ Log-normal standard deviation $\sigma_{\text {durbad }}$} \\
\hline 85 & 0.83 & 0.88 & 0.88 & 0.84 & 0.83 & 0.83 & 0.85 & 0.80 \\
\hline 80 & 0.84 & 0.88 & 0.93 & 0.88 & 0.84 & 0.84 & 0.90 & 0.86 \\
\hline 75 & 0.85 & 0.93 & 0.97 & 0.92 & 0.88 & 0.91 & 0.93 & 0.90 \\
\hline 70 & 0.82 & 0.96 & 1.06 & 0.99 & 0.86 & 0.94 & 0.97 & 0.93 \\
\hline 65 & 0.85 & 1.02 & 1.11 & 1.04 & 0.87 & 1.00 & 1.13 & 0.97 \\
\hline 60 & 0.86 & 1.08 & 1.17 & 1.10 & 0.88 & 1.08 & 1.23 & 1.03 \\
\hline 55 & 0.89 & 1.13 & 1.17 & 1.15 & 0.92 & 1.14 & 1.21 & 1.10 \\
\hline 50 & 0.94 & 1.17 & 1.20 & 1.18 & 0.93 & 1.17 & 1.20 & 1.14 \\
\hline 45 & 0.99 & 1.21 & 1.21 & 1.21 & 0.97 & 1.23 & 1.20 & 1.19 \\
\hline 40 & 1.04 & 1.26 & 1.26 & 1.26 & 1.02 & 1.25 & 1.23 & 1.23 \\
\hline 35 & 1.10 & 1.31 & 1.26 & 1.29 & 1.07 & 1.28 & 1.25 & 1.27 \\
\hline 30 & 1.20 & 1.37 & 1.34 & 1.34 & 1.11 & 1.31 & 1.32 & 1.36 \\
\hline 25 & 1.28 & 1.47 & 1.39 & 1.42 & 1.19 & 1.36 & 1.33 & 1.40 \\
\hline 20 & 1.39 & 1.56 & 1.45 & 1.52 & 1.29 & 1.40 & 1.38 & 1.48 \\
\hline 15 & 1.52 & 1.67 & 1.56 & 1.67 & 1.46 & 1.53 & 1.47 & 1.61 \\
\hline \multicolumn{9}{|c|}{ Log-normal mean $\mu_{\text {dur,good }}$} \\
\hline 85 & 3.89 & 3.84 & 3.77 & 3.86 & 3.96 & 4.10 & 4.08 & 4.13 \\
\hline 80 & 3.95 & 3.69 & 3.61 & 3.77 & 3.92 & 4.09 & 4.13 & 4.13 \\
\hline 75 & 4.06 & 3.54 & 3.30 & 3.53 & 4.03 & 4.19 & 4.16 & 4.25 \\
\hline 70 & 3.91 & 3.25 & 2.98 & 3.37 & 3.98 & 4.12 & 3.96 & 4.12 \\
\hline 65 & 3.90 & 3.06 & 2.77 & 3.21 & 3.95 & 3.86 & 3.58 & 3.93 \\
\hline 60 & 3.88 & 2.88 & 2.60 & 3.08 & 3.87 & 3.66 & 3.51 & 3.77 \\
\hline 55 & 3.84 & 2.68 & 2.45 & 2.90 & 3.82 & 3.46 & 3.18 & 3.69 \\
\hline 50 & 3.78 & 2.52 & 2.30 & 2.75 & 3.78 & 3.26 & 2.93 & 3.50 \\
\hline 45 & 3.66 & 2.38 & 2.21 & 2.60 & 3.70 & 3.04 & 2.69 & 3.26 \\
\hline 40 & 3.53 & 2.25 & 2.03 & 2.46 & 3.62 & 2.82 & 2.50 & 3.06 \\
\hline 35 & 3.32 & 2.08 & 1.92 & 2.30 & 3.52 & 2.59 & 2.38 & 2.85 \\
\hline 30 & 3.08 & 1.88 & 1.81 & 2.18 & 3.39 & 2.36 & 2.16 & 2.57 \\
\hline 25 & 2.79 & 1.72 & 1.76 & 2.04 & 3.20 & 2.17 & 1.98 & 2.33 \\
\hline 20 & 2.48 & 1.54 & 1.64 & 1.89 & 2.94 & 1.99 & 1.82 & 2.07 \\
\hline 15 & 2.16 & 1.36 & 1.50 & 1.65 & 2.71 & 1.80 & 1.61 & 1.76 \\
\hline
\end{tabular}


TABLE 2: Continued.

\begin{tabular}{lcccccccc}
\hline Elevation & Front & Front-right & Right & Back-right & Back & Back-left & Left & Front-left \\
\hline & & & Log-normal standard deviation $\sigma_{\text {dur,good }}$ & & \\
85 & 1.78 & 1.71 & 1.73 & 1.70 & 1.72 & 1.70 & 1.81 \\
80 & 1.70 & 1.69 & 1.65 & 1.61 & 1.75 & 1.82 & 1.82 \\
75 & 1.67 & 1.64 & 1.62 & 1.67 & 1.74 & 1.78 & 1.81 \\
70 & 1.75 & 1.66 & 1.63 & 1.62 & 1.75 & 1.93 & 1.89 \\
65 & 1.77 & 1.63 & 1.56 & 1.59 & 1.77 & 1.98 & 1.87 \\
60 & 1.78 & 1.58 & 1.49 & 1.55 & 1.82 & 1.90 & 1.75 \\
55 & 1.77 & 1.54 & 1.44 & 1.50 & 1.79 & 1.83 & 1.74 \\
50 & 1.77 & 1.49 & 1.38 & 1.45 & 1.81 & 1.78 & 1.97 \\
45 & 1.75 & 1.43 & 1.31 & 1.40 & 1.80 & 1.72 & 1.85 \\
40 & 1.78 & 1.36 & 1.27 & 1.35 & 1.79 & 1.66 & 1.58 \\
35 & 1.81 & 1.32 & 1.20 & 1.29 & 1.77 & 1.58 & 1.51 \\
30 & 1.81 & 1.28 & 1.20 & 1.23 & 1.73 & 1.50 & 1.43 \\
25 & 1.78 & 1.23 & 1.14 & 1.18 & 1.67 & 1.39 & 1.36 \\
20 & 1.71 & 1.18 & 1.12 & 1.12 & 1.60 & 1.30 & 1.26 \\
15 & 1.54 & 1.11 & 1.09 & 1.07 & 1.51 & 1.21 & 1.22 \\
\hline
\end{tabular}

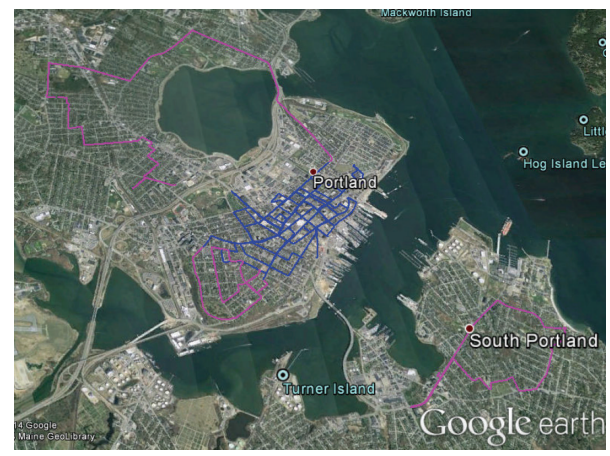

Figure 4: Measurement route in Portland (Maine) across urban (blue) and suburban (magenta) environment.

satellites of Sirius Satellite Radio were recorded at the operational frequency of $2.3 \mathrm{GHz}$ (S-Band) with a sampling rate of $2.1 \mathrm{kHz}$. The precise measuring time and vehicle position was logged via GPS. In addition, a hemispheric camera was mounted on the van to document the environment, pointing towards the sky ( 5 frames per second with a resolution of 880 $\times 768$ pixels of the fisheye region). The campaign was carried out along a distance of $3700 \mathrm{~km}$ from Jacksonville (Florida) to Portland (Maine) in different environments (urban, suburban, commercial, and highway). In this contribution we focus on data from Portland (Maine) comprising urban and suburban environment. Figure 4 illustrates the measurement route in urban and suburban environments (depicted by the different colors) in Portland, Maine.

3.3. Evaluation of the Image-Based State Estimation Method. In order to validate the image-based method we compare the estimated channel states of the images with actual radio frequency (RF) Data.

In LMS channel models the sequence of states is commonly modeled by Markov/semi-Markov processes [1,37].
Therefore, parameters like state probabilities and the distribution of state durations are of interest. The state duration statistics are derived for the satellites Sirius 1, Sirius 2, XM3 , and XM- 4 with an average elevation of $65^{\circ}, 68^{\circ}, 38^{\circ}$, and $23^{\circ}$, respectively. The results based on image and RF data are depicted in Figure 5 for bad state and in Figure 6 for good state. In order to evaluate the estimation accuracy, we show the Complementary Cumulative Distribution Function (CCDF).

The statistics show very good agreement between the RFbased (dashed lines) and the image-based results (solid lines). Additionally, the log-normal fit is plotted in black. As a result, the image-based approach can be seen as a valid technique for state estimation.

\section{Image-Based Model of the Land Mobile Satellite Channel}

In the following we explain the proposed LMS channel model. Statistical channel models reproduce the time-series of the received satellite signal comprising two processes, the very slow fading and the slow/fast fading. The nonstationarity of the signal due to the different degrees of shadowing is expressed by states (e.g., LoS, moderate shadowing, and deep shadowing). Well accepted in the literature are LMS models, which describe the very slow fading component by either three states [1], namely, line-of-sight, shadowed, and blocked, or by two states [2], good and bad. The slow and fast fading caused by varying shadowing and multipath effects are treated as a second process and is commonly reproduced by a stationary stochastic process.

In Figure 7, the LMS model is illustrated, which is based on the versatile two-state approach presented in $[2,5]$. The model distinguishes between two states, good and bad, corresponding to LoS or light shadowing and NLoS or heavy shadowing, respectively. The state modeling is performed by 
TABLE 3: Semi-Markov parameters (good and bad state) for suburban environment for variable driving directions and elevations.

\begin{tabular}{|c|c|c|c|c|c|c|c|c|}
\hline Elevation & Front & Front-right & Right & Back-right & Back & Back-left & Left & Front-left \\
\hline \multicolumn{9}{|c|}{ Log-normal mean $\mu_{\text {dur,bad }}$} \\
\hline 85 & 1.87 & 1.83 & 1.88 & 1.81 & 1.81 & 1.79 & 1.81 & 1.82 \\
\hline 80 & 1.85 & 1.88 & 1.89 & 1.86 & 1.81 & 1.78 & 1.78 & 1.82 \\
\hline 75 & 1.89 & 1.94 & 1.96 & 1.86 & 1.78 & 1.78 & 1.78 & 1.82 \\
\hline 70 & 1.92 & 1.95 & 1.93 & 1.91 & 1.79 & 1.75 & 1.75 & 1.80 \\
\hline 65 & 1.89 & 1.97 & 1.96 & 1.93 & 1.79 & 1.79 & 1.79 & 1.80 \\
\hline 60 & 1.91 & 2.02 & 1.99 & 1.96 & 1.83 & 1.82 & 1.88 & 1.83 \\
\hline 55 & 1.94 & 2.04 & 2.01 & 2.02 & 1.85 & 1.86 & 1.90 & 1.86 \\
\hline 50 & 1.97 & 2.06 & 1.98 & 2.05 & 1.90 & 1.88 & 1.89 & 1.91 \\
\hline 45 & 1.98 & 2.05 & 1.94 & 2.04 & 1.94 & 1.90 & 1.92 & 1.90 \\
\hline 40 & 2.04 & 2.07 & 1.95 & 2.07 & 1.99 & 1.95 & 1.94 & 1.98 \\
\hline 35 & 2.07 & 2.10 & 2.01 & 2.08 & 2.04 & 1.99 & 1.98 & 1.97 \\
\hline 30 & 2.13 & 2.14 & 2.10 & 2.15 & 2.11 & 2.03 & 1.99 & 1.99 \\
\hline 25 & 2.10 & 2.22 & 2.19 & 2.24 & 2.19 & 2.09 & 2.07 & 2.07 \\
\hline 20 & 2.15 & 2.34 & 2.32 & 2.42 & 2.26 & 2.22 & 2.19 & 2.14 \\
\hline 15 & 2.38 & 2.57 & 2.55 & 2.65 & 2.41 & 2.41 & 2.29 & 2.35 \\
\hline \multicolumn{9}{|c|}{ Log-normal standard deviation $\sigma_{\text {dur,bad }}$} \\
\hline 85 & 0.92 & 0.95 & 0.93 & 0.93 & 0.94 & 0.96 & 0.97 & 0.97 \\
\hline 80 & 0.97 & 0.96 & 0.95 & 0.93 & 0.95 & 0.98 & 0.96 & 0.96 \\
\hline 75 & 0.95 & 0.97 & 0.95 & 0.98 & 0.96 & 0.95 & 0.95 & 0.93 \\
\hline 70 & 0.95 & 0.98 & 0.98 & 0.99 & 0.97 & 0.95 & 0.99 & 0.95 \\
\hline 65 & 0.98 & 1.00 & 0.96 & 0.99 & 1.00 & 0.95 & 1.00 & 0.98 \\
\hline 60 & 1.00 & 0.99 & 0.96 & 1.00 & 1.00 & 0.98 & 0.97 & 0.99 \\
\hline 55 & 0.98 & 1.01 & 0.97 & 0.99 & 1.00 & 0.97 & 0.98 & 1.00 \\
\hline 50 & 1.01 & 1.03 & 0.98 & 0.98 & 1.00 & 0.98 & 1.00 & 0.99 \\
\hline 45 & 1.06 & 1.07 & 1.02 & 1.02 & 1.02 & 1.03 & 1.00 & 1.04 \\
\hline 40 & 1.09 & 1.08 & 1.03 & 1.05 & 1.04 & 1.04 & 1.01 & 1.05 \\
\hline 35 & 1.14 & 1.10 & 1.02 & 1.09 & 1.08 & 1.09 & 1.02 & 1.10 \\
\hline 30 & 1.18 & 1.12 & 1.06 & 1.12 & 1.13 & 1.13 & 1.07 & 1.14 \\
\hline 25 & 1.27 & 1.18 & 1.13 & 1.18 & 1.21 & 1.18 & 1.08 & 1.18 \\
\hline 20 & 1.36 & 1.27 & 1.24 & 1.26 & 1.31 & 1.21 & 1.10 & 1.26 \\
\hline 15 & 1.47 & 1.45 & 1.38 & 1.47 & 1.41 & 1.30 & 1.21 & 1.35 \\
\hline \multicolumn{9}{|c|}{ Log-normal mean $\mu_{\text {dur,good }}$} \\
\hline 85 & 2.94 & 2.93 & 2.99 & 2.92 & 2.94 & 2.97 & 2.98 & 2.93 \\
\hline 80 & 2.98 & 2.96 & 2.98 & 2.95 & 2.96 & 2.95 & 2.93 & 2.95 \\
\hline 75 & 3.00 & 2.99 & 2.98 & 2.94 & 2.90 & 2.93 & 2.90 & 2.97 \\
\hline 70 & 2.99 & 2.93 & 2.87 & 2.94 & 2.93 & 2.84 & 2.87 & 2.96 \\
\hline 65 & 2.92 & 2.88 & 2.78 & 2.91 & 2.90 & 2.86 & 2.91 & 2.92 \\
\hline 60 & 2.92 & 2.80 & 2.68 & 2.84 & 2.89 & 2.92 & 2.91 & 2.92 \\
\hline 55 & 2.89 & 2.71 & 2.59 & 2.77 & 2.89 & 2.94 & 2.90 & 2.91 \\
\hline 50 & 2.87 & 2.61 & 2.49 & 2.68 & 2.89 & 2.89 & 2.81 & 2.90 \\
\hline 45 & 2.80 & 2.51 & 2.36 & 2.59 & 2.87 & 2.83 & 2.69 & 2.81 \\
\hline 40 & 2.76 & 2.40 & 2.24 & 2.52 & 2.90 & 2.75 & 2.59 & 2.82 \\
\hline 35 & 2.71 & 2.29 & 2.08 & 2.40 & 2.87 & 2.68 & 2.48 & 2.72 \\
\hline 30 & 2.65 & 2.13 & 1.96 & 2.26 & 2.83 & 2.57 & 2.33 & 2.56 \\
\hline 25 & 2.50 & 1.93 & 1.78 & 2.08 & 2.80 & 2.40 & 2.18 & 2.41 \\
\hline 20 & 2.30 & 1.75 & 1.65 & 1.91 & 2.69 & 2.22 & 1.98 & 2.19 \\
\hline 15 & 2.15 & 1.55 & 1.52 & 1.69 & 2.38 & 1.99 & 1.73 & 1.93 \\
\hline
\end{tabular}


TABLE 3: Continued.

\begin{tabular}{lcccccccc}
\hline Elevation & Front & Front-right & Right & Back-right & Back & Back-left & Left & Front-left \\
\hline 85 & & & Log-normal standard deviation $\sigma_{\text {dur,good }}$ & & \\
80 & 1.49 & 1.49 & 1.46 & 1.46 & 1.49 & 1.49 & 1.50 \\
75 & 1.47 & 1.44 & 1.41 & 1.44 & 1.49 & 1.52 & 1.51 \\
70 & 1.47 & 1.39 & 1.34 & 1.43 & 1.52 & 1.53 & 1.55 \\
65 & 1.48 & 1.36 & 1.30 & 1.38 & 1.53 & 1.59 & 1.56 \\
60 & 1.51 & 1.31 & 1.22 & 1.33 & 1.55 & 1.58 & 1.51 \\
55 & 1.50 & 1.28 & 1.22 & 1.30 & 1.58 & 1.57 & 1.52 \\
50 & 1.48 & 1.26 & 1.19 & 1.28 & 1.57 & 1.50 & 1.46 \\
45 & 1.49 & 1.25 & 1.17 & 1.25 & 1.58 & 1.48 & 1.41 \\
40 & 1.54 & 1.25 & 1.15 & 1.20 & 1.57 & 1.47 & 1.55 \\
35 & 1.55 & 1.25 & 1.13 & 1.18 & 1.52 & 1.41 & 1.36 \\
30 & 1.57 & 1.19 & 1.06 & 1.17 & 1.52 & 1.35 & 1.31 \\
25 & 1.57 & 1.14 & 1.01 & 1.13 & 1.52 & 1.30 & 1.28 \\
20 & 1.57 & 1.11 & 1.00 & 1.09 & 1.51 & 1.27 & 1.24 \\
15 & 1.55 & 1.03 & 0.97 & 1.05 & 1.46 & 1.53 & 1.17 \\
\hline
\end{tabular}

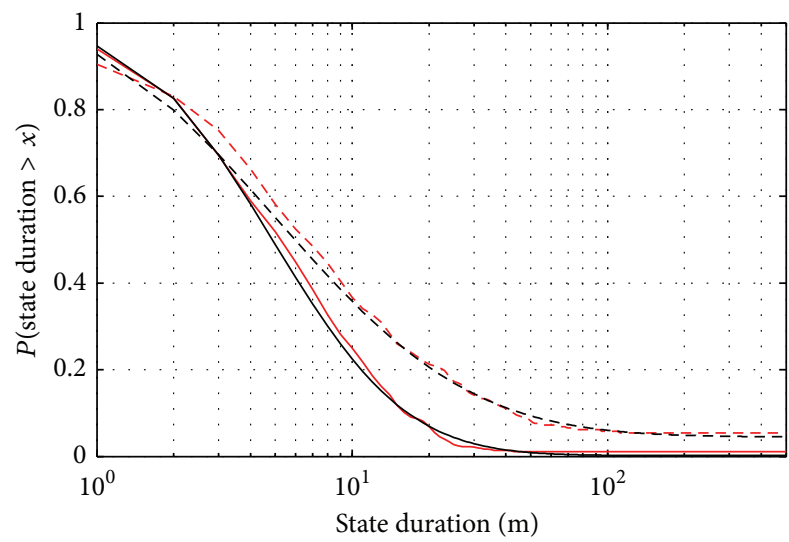

(a) Sirius 1

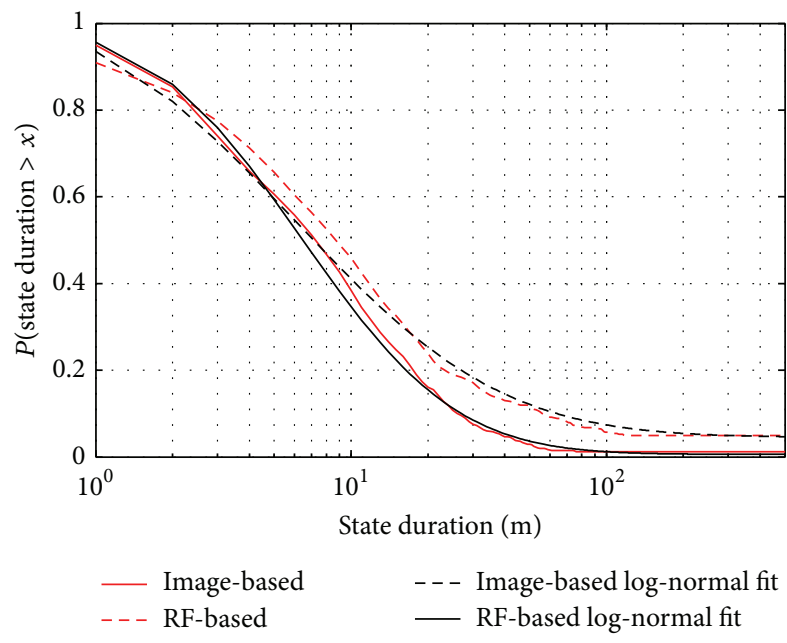

(c) $\mathrm{XM}-3$

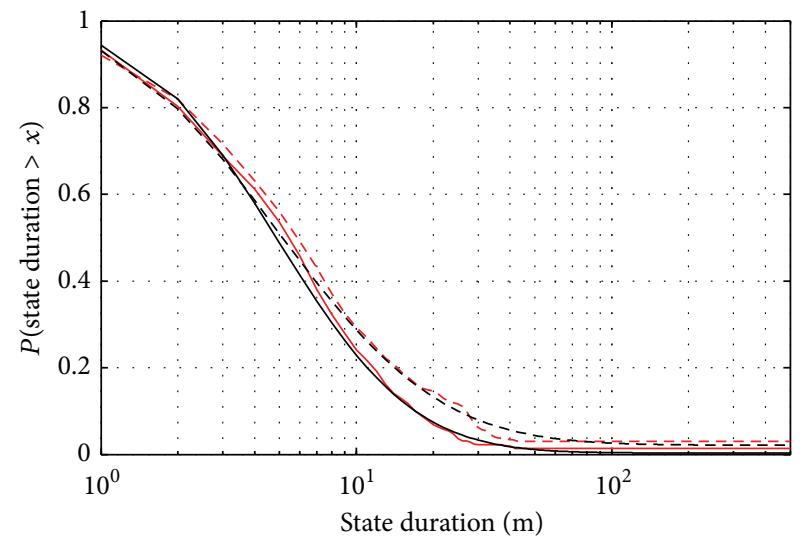

(b) Sirius 2

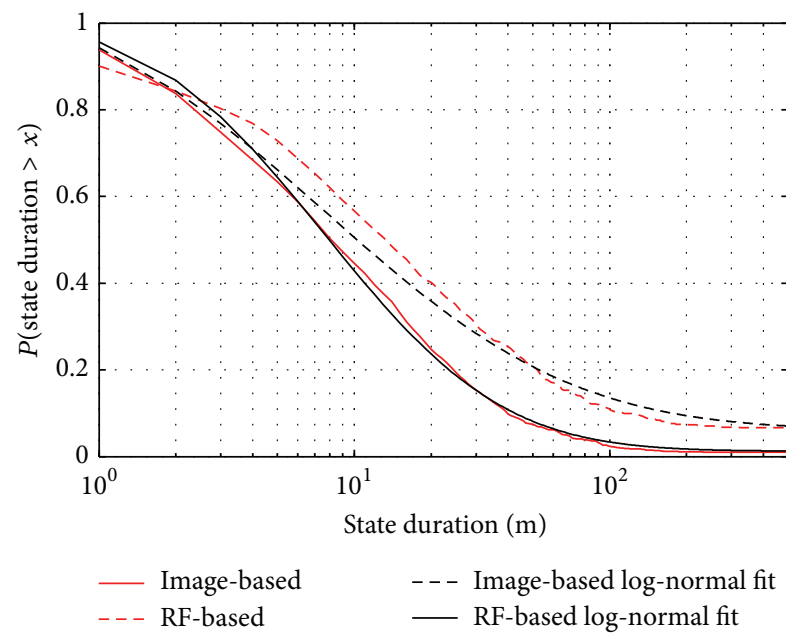

(d) XM-4

FIGURE 5: Statistics for bad state for each satellite (Sirius 1, Sirius 2, XM-3, and XM-4) obtained from the image-based state estimation method (red solid lines). As a ground truth, the same plots are shown for the states obtained from the RF measurements (red dashed lines). Furthermore, the log-normal fitting is shown based on images and RF data, respectively (black curves). 


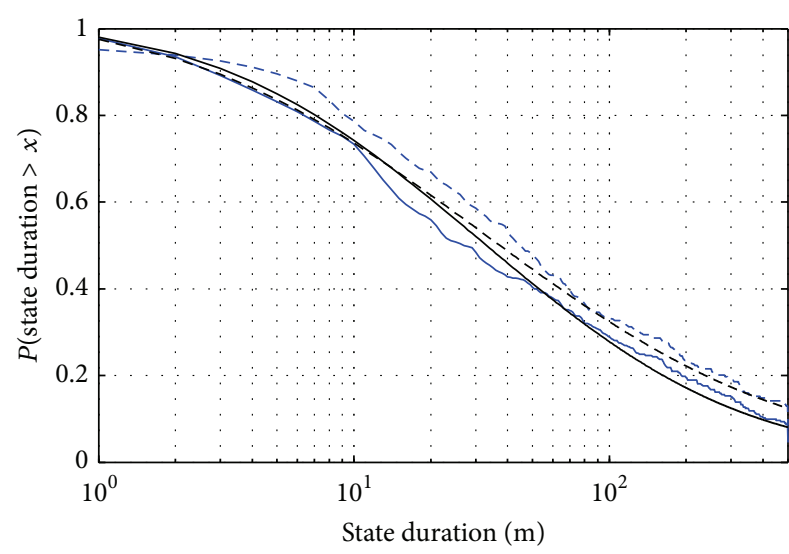

(a) Sirius 1

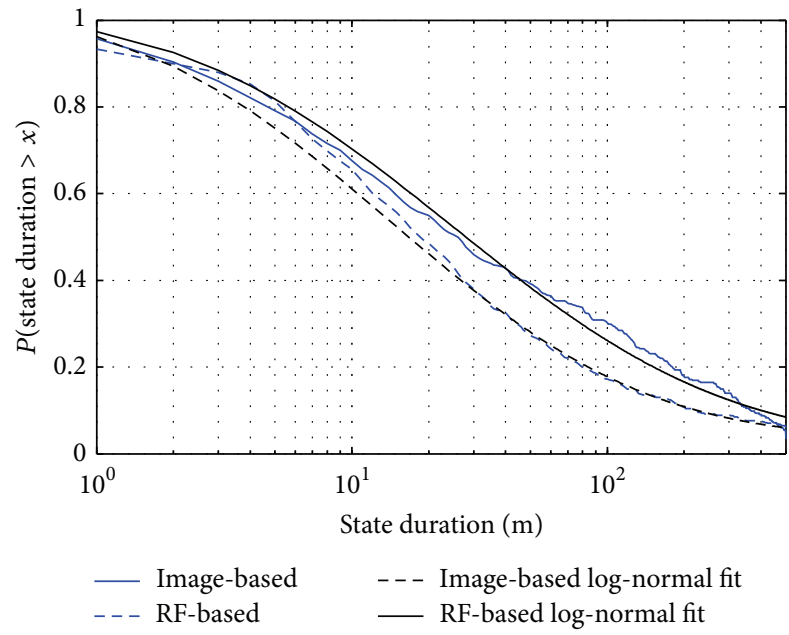

(c) XM-3

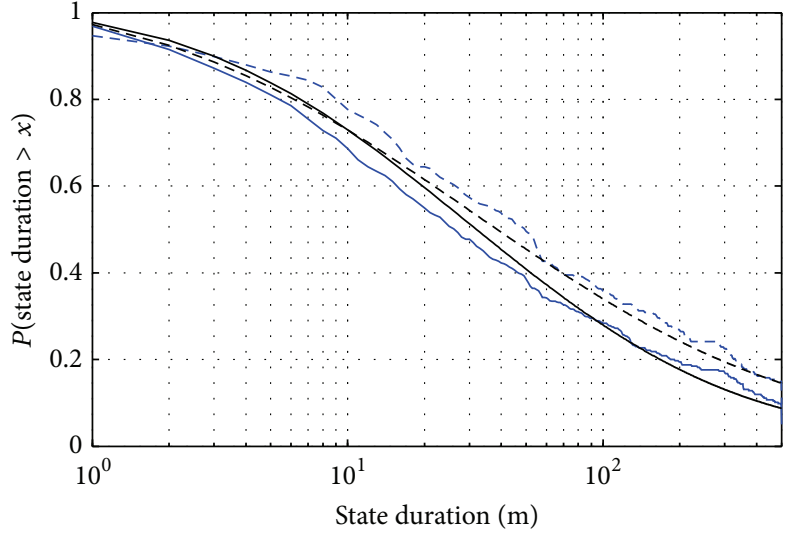

(b) Sirius 2

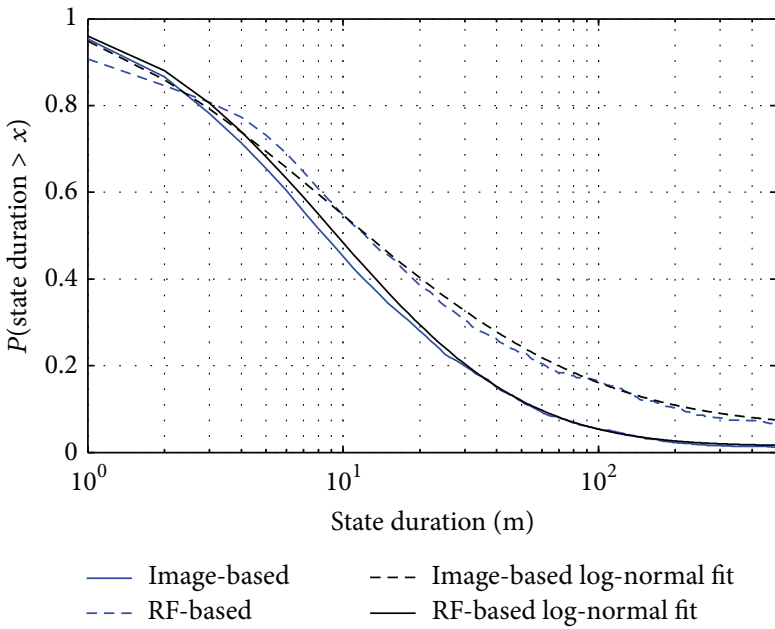

(d) XM-4

FIGURE 6: Statistics for good state for each satellite (Sirius 1, Sirius 2, XM-3, and XM-4) obtained from the image-based state estimation method (blue solid lines). As a ground truth, the same plots are shown for the states obtained from the RF measurements (blue dashed lines). Furthermore, the log-normal fitting is shown based on images and RF data, respectively (black curves).

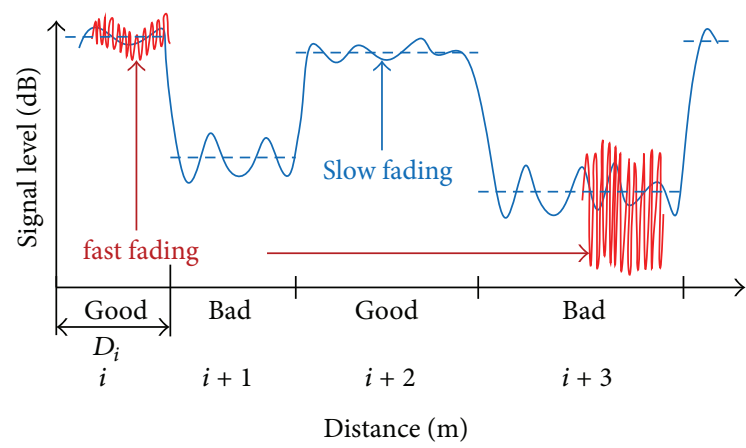

FIgURE 7: Features of the proposed two-state LMS model, where $i$ is the state interval index. Each state has a possibly different interval duration $D_{i}$ and a new Loo triplet $\Omega_{i}$ consisting of $M_{A, i}, \Sigma_{A, i}$, and $M P_{i}$ drawn from random processes.

a semi-Markov chain [37], which improves the accuracy of the state duration modeling, compared to the more common first order Markov chains [1]. Based on the measured state durations, the state duration probability density function (SDPDF) is modeled by a log-normal distribution. It is expressed by

$$
P(D)=\frac{1}{D \sigma_{\text {dur }} \sqrt{2 \pi}} \exp \left(-\frac{\left(\ln (D)-\mu_{\mathrm{dur}}\right)^{2}}{2 \sigma_{\text {dur }}^{2}}\right),
$$

where $D$ is the state duration, $\mu_{\text {dur }}$ is the mean value of $\ln (D)$, and $\sigma_{\text {dur }}$ is the standard deviation of $\ln (D)$. These parameters $\mu_{\text {dur }}$ and $\sigma_{\text {dur }}$ are called the log-normal mean and log-normal standard deviation, respectively, and are estimated for both good and bad state. The mean of the state duration (in meter) can be calculated by $\mathrm{e}^{\mu_{\mathrm{dur}}+\sigma_{\text {dur }}^{2} / 2}$; the standard deviation (in

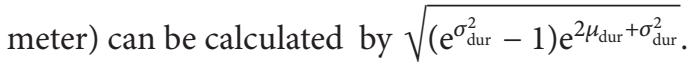

The slow and fast signal fading within each state are modeled by a Loo distribution [27]. The Loo distribution considers the received signal as a sum of two signal components. A log-normally distributed direct signal expresses the slow fading component corresponding to varying shadowing 


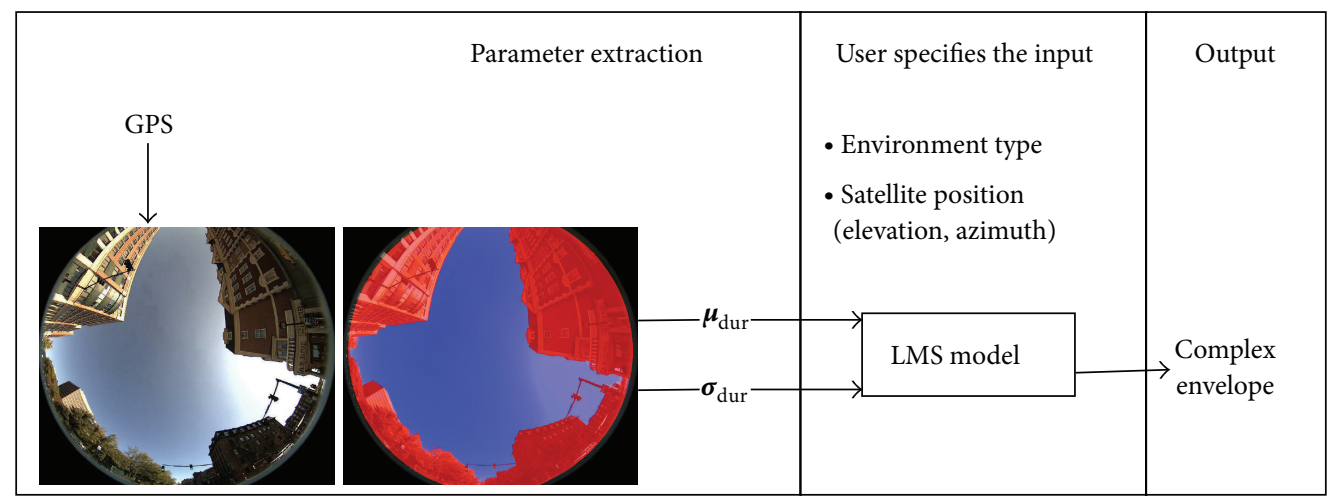

FIGURE 8: High-level overview of the proposed image-based LMS model. The image-based state duration parameters $\boldsymbol{\mu}_{\mathrm{dur}}$ and $\boldsymbol{\sigma}_{\mathrm{dur}}$ are preprocessed for all possible environments and satellite positions. Depending on the user's input, that is, environment type and satellite position, the specific $\mu_{\text {dur }}$ and $\sigma_{\text {dur }}$ are chosen.
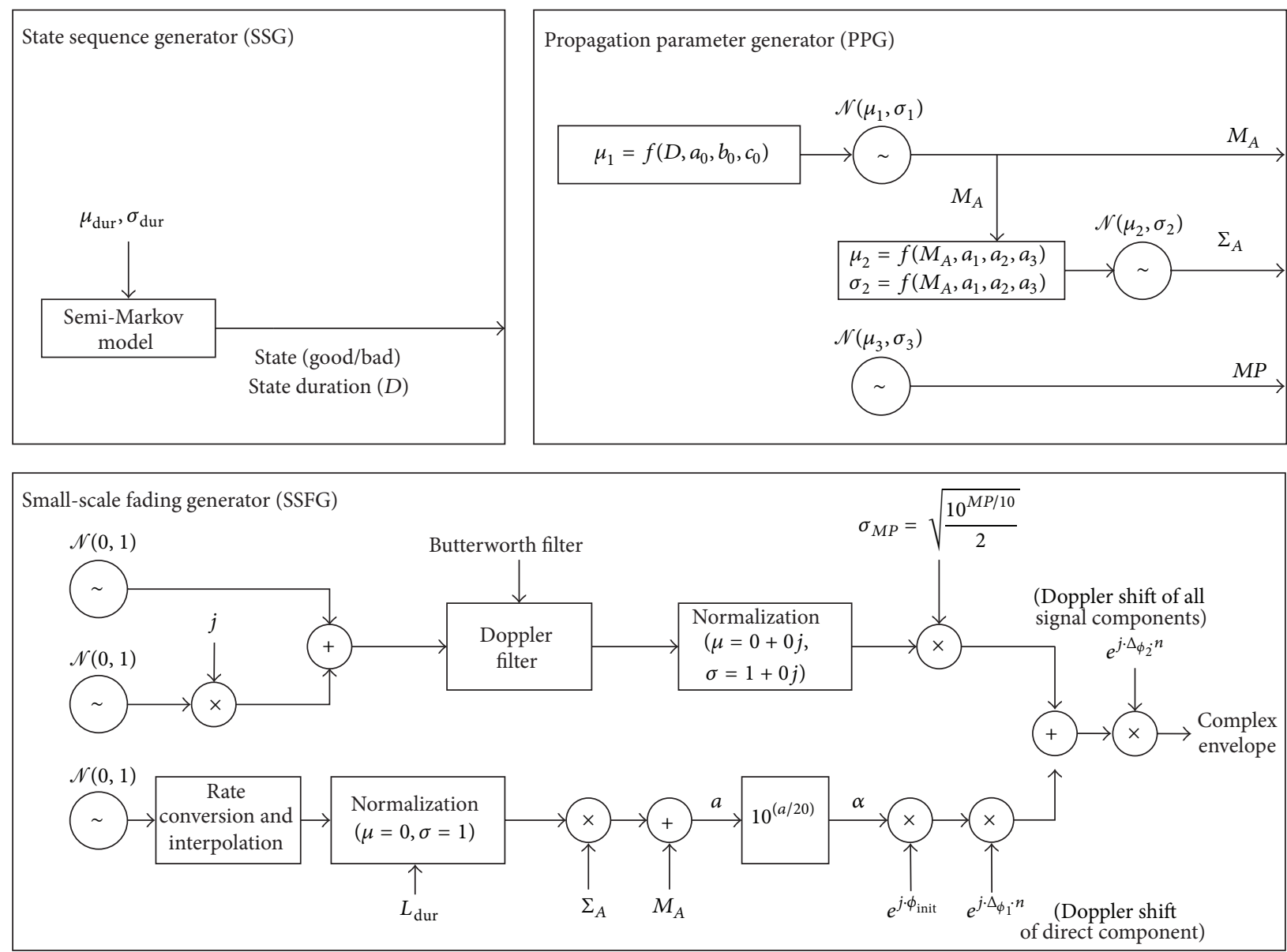

FIGURE 9: Detailed LMS model implementation diagram comprising the three main elements: state sequence generator (SSG), propagation parameter generator (PPG), and small-scale fading generator (SSFG).

conditions of the direct signal. A Rice distribution characterizes the fast fading component due to multipath effects. The Loo parameter triplet $\Omega$ consists of the mean $M_{A}$, the standard deviation $\Sigma_{A}$ for the log-normally distributed direct signal, and the average multipath power MP. Depending on the current state interval $i$ and on the environment of the terminal, a new random Loo parameter triplet $\boldsymbol{\Omega}_{i}=$ $\left(M_{A, i}, \Sigma_{A, i}, M P_{i}\right)$ is generated. Compared to an earlier threestate model [1], where the Loo parameter triplet is fixed for each state, the versatile Loo parameter selection enables 


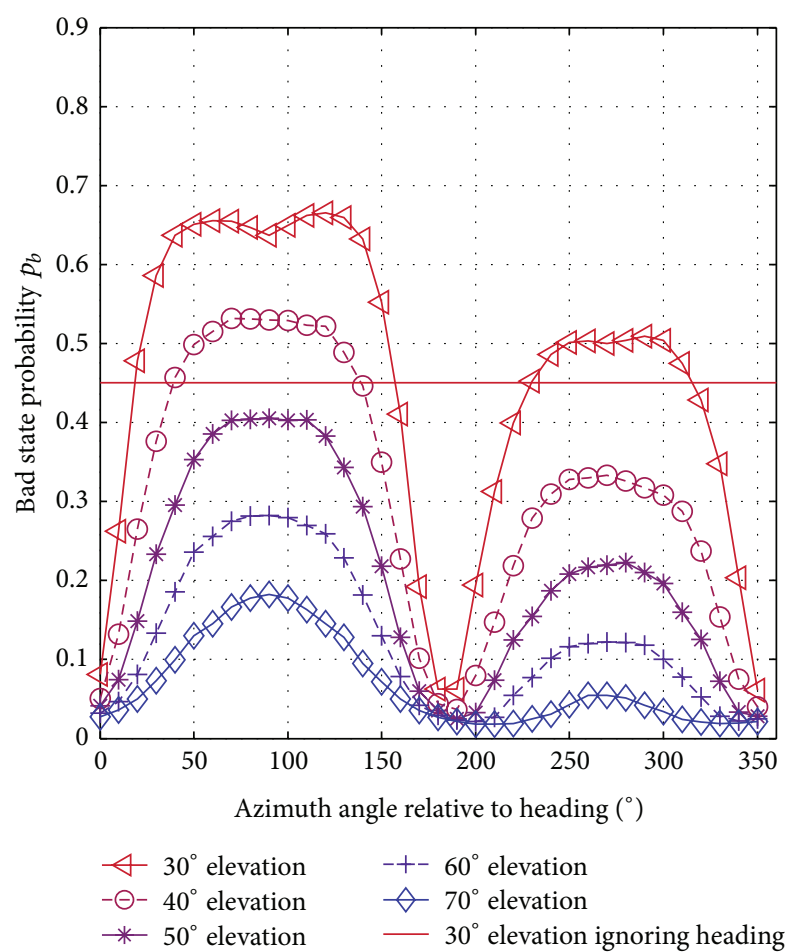

(a) Urban environment

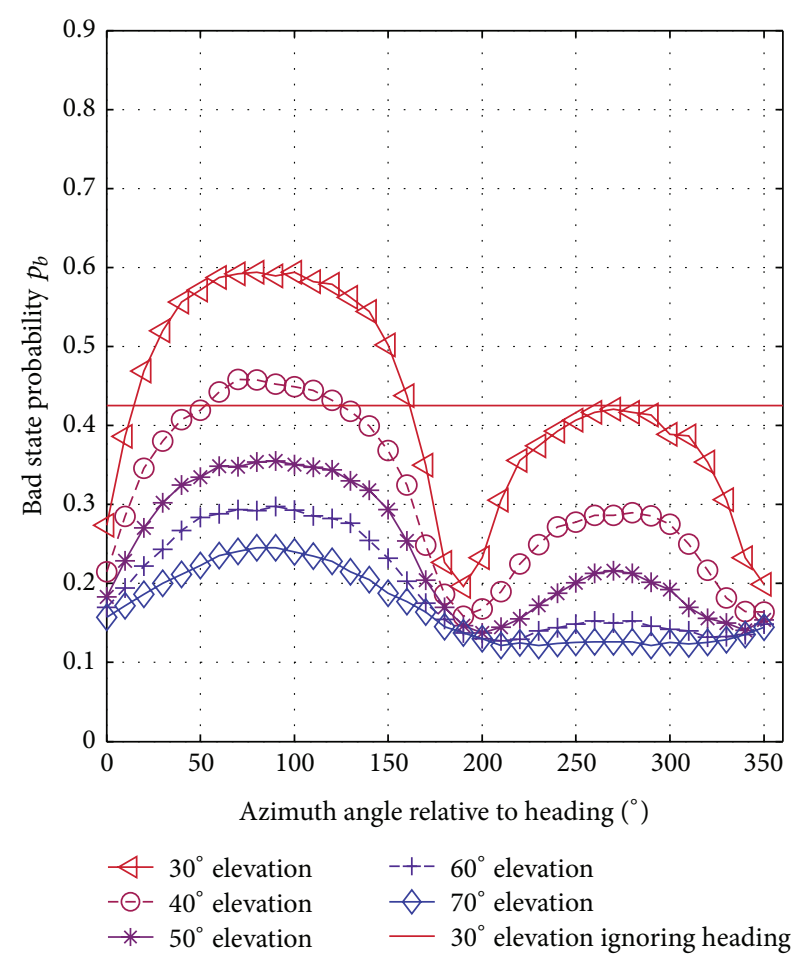

(b) Suburban environment

FIgURE 10: The bad state probability (service unavailability) for urban (a) and suburban (b) environment. The dependency on the azimuth angle (relative to driving direction) is shown for satellites at different elevation angles. For an elevation of $30^{\circ}$ also the result of ignoring the heading is plotted as a constant (red line).

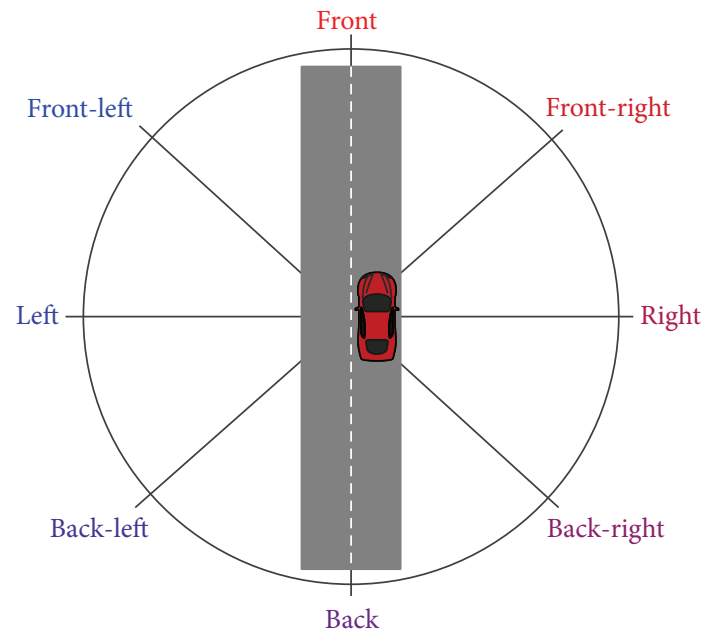

FIGURE 11: Eight exemplary satellite positions relative to the street orientation, which are further investigated.

a more realistic modeling over the full dynamic range of the received signal [2]. The output of the channel model is a timeseries of the received signal in form of a complex envelope. For the purpose of comparability, we convert the output to a time-series of the carrier-to-noise ratio $(C / N)$. As the LoS level is dependent on the transmit power, path-loss (distance, weather, and frequency), and the receiver characteristics, we remove it from the signal to obtain a representation of the fading effects exclusively caused by the environment. Thus, we end up with a carrier-to-noise ratio normalized to the LoS level $C / N-(C / N)_{\text {LoS }}$ in $\mathrm{dB}$.

Figure 8 shows how the parameters of the model are preprocessed and how they are applied. First, hemispheric images are binarized into the classes sky and obstruction. Second, a set of parameters for the state duration statistics is extracted from the images, depending on the environment type, satellite elevation, and satellite azimuth. Depending on the user's needs, representative parameters are selected for the actual LMS channel modeling process. Despite the extracted state duration parameters (very slow fading), the Loo-parameters (slow and fast fading) are chosen. Finally, a time-series of the LMS channel in terms of the complex envelope is simulated.

In Figure 9 an overview of the detailed implementation of the LMS channel model is depicted. The model is structured in three different generator types [5].

(1) The State Sequence Generator (SSG) reproduces as sequence of good and bad states by a semi-Markov model process. The necessary parameters are the lognormal mean $\mu_{\text {dur }}$ and log-normal standard deviation $\sigma_{\text {dur }}$ of the state duration distribution for the good and bad state, respectively (cf. (1)). With this approach, an accurate modeling of the state duration and the state probabilities can be provided. At the $i$-th interval, 


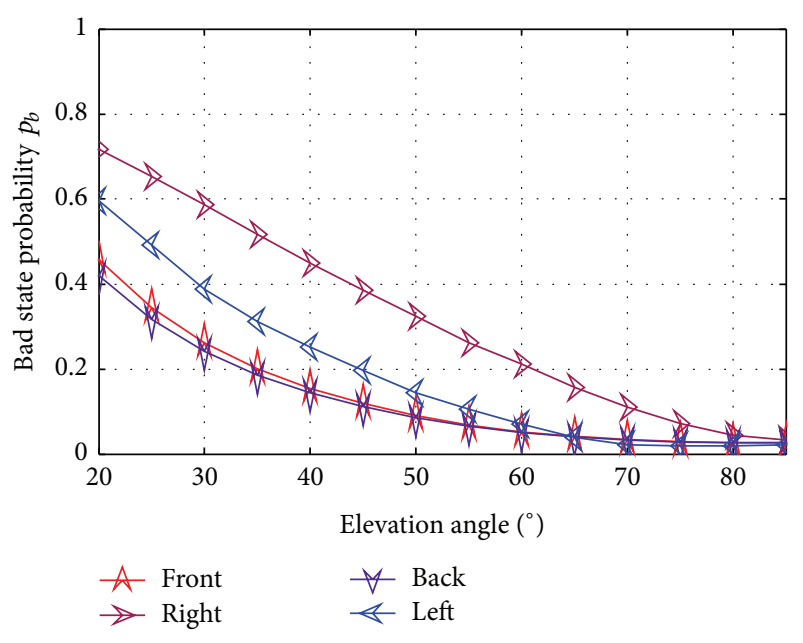

(a) Urban environment

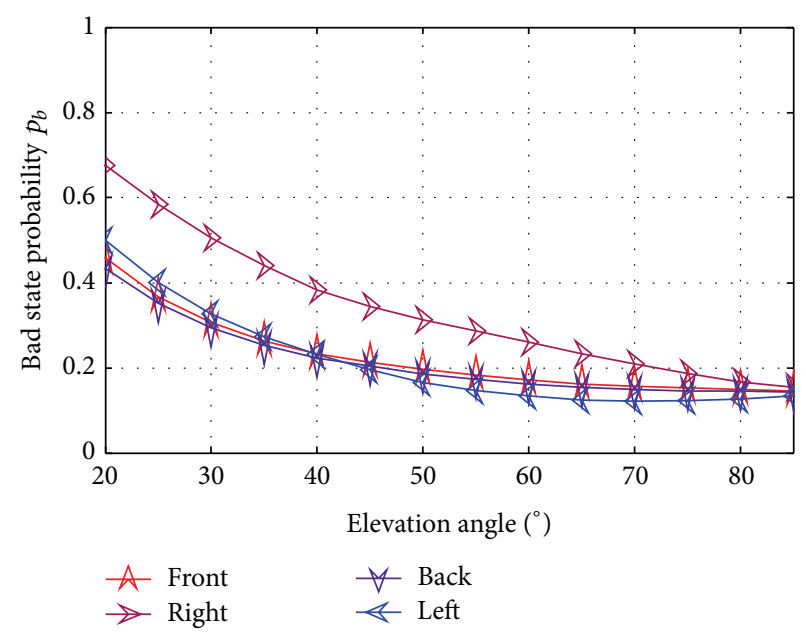

(b) Subrban environment

Figure 12: Bad state probability $p_{b}$ for different driving directions in urban (a) and suburban (b) environment.

the SSG outputs the current state, which can be either good or bad, and the duration $D_{i}$ for which the channel remains in the same state. The lognormal state duration parameters $\left(\mu_{\text {dur }}, \sigma_{\text {dur }}\right)$, as input parameters of the semi-Markov model, are provided in Tables 2 and 3.

(2) The Propagation Parameter Generator (PPG) is invoked at each new state interval producing a new random Loo triplet $\boldsymbol{\Omega}_{i}$. As depicted in Figure 9, the PPG features three independent standard normal generators, whose means and variances $\left(\mu_{1}, \sigma_{1}, \mu_{2}, \sigma_{2}, \mu_{3}, \sigma_{3}\right)$ are estimated from measured $\mathrm{RF}$ data by curve-fitting as presented in [38].

(3) The Small-Scale Fading Generator (SSFG) computes the Loo distributed time-series including Doppler shaping for every new state interval, which is the output of the proposed LMS channel model. Furthermore, a Doppler shift of the log-normal varying direct signal is included, which is related to the speed of the vehicle. An optional Doppler shift of all signal components is also possible, to include the movement of the satellite.

\section{Results}

In the following we show the results obtained by the LMS channel model explained in Section 4, where the model parameters have been obtained with the image-based state estimation presented in Section 3. The first part of this section focuses on the state parameters of the model, which are important for a reliable state sequence generation. Secondly, the output of the whole channel model is evaluated in terms of first order statistics.
Exploiting the image-based state parameter estimation (instead of RF measurements) offers the advantage to perform a reliable analysis of the impact of the driving direction. Without any further geometrical consideration, a statistical availability prediction depending on the direction of the road is possible.

One crucial parameter of satellite service prediction is the service unavailability, or so-called bad state probability $p_{b}$. Figure 10 depicts $p_{b}$ with respect to different elevation angles, azimuth angles (one degree resolution), and environment types (urban in Figure 10(a) and suburban in Figure 10(b)). The different curves represent different elevation angles, from which an inverse proportionality of $p_{b}$ against the elevation angle can be seen. Moreover, a nonsymmetric dependency on the azimuth angle relative to the heading (abscissa) is visible. In both plots two maxima can be identified, one global at $90^{\circ}$ azimuth and one local at $270^{\circ}$ azimuth, which correspond to the right side and left side of the vehicle, respectively. The global maximum is caused by the right-hand driving on the street; therefore obstacles at the right side of the vehicle appear to be higher in relation to the position of the terminal on the street. This result shows that an assumption of the symmetric dependency of the azimuth angle is an oversimplification. At $0^{\circ}$ and $180^{\circ}$, corresponding to front and back of the vehicle, two minima are found. This clearly reflects the street canyon characteristics, especially in urban scenarios. The influence of the street canyon decreases with an increasing elevation angle. The constant (solid red line) results from a calculation of $p_{b}$, which ignores the heading of the vehicle (exemplary for an elevation angle of $30^{\circ}$ ). Here, the heading is assumed to be uniformly distributed, representing the state-of-the-art LMS channel models, which do not include the azimuth angle of the satellite, for example, [2]. Ignoring the driving direction leads to a significant overestimation or underestimation of the bad state probability $p_{b}$. The difference between urban (Figure 10(a)) and suburban environment (Figure 10(b)) 


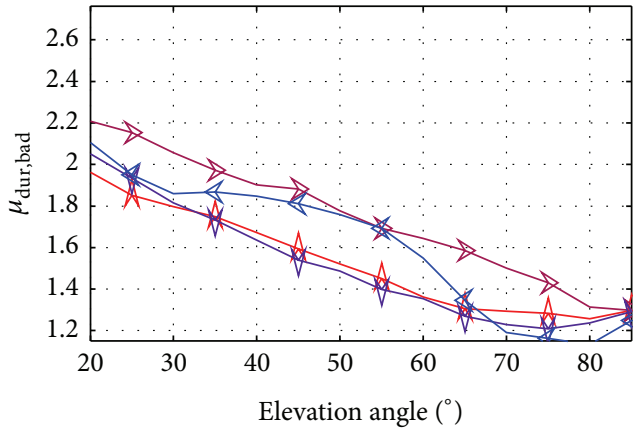

(a) Urban environment

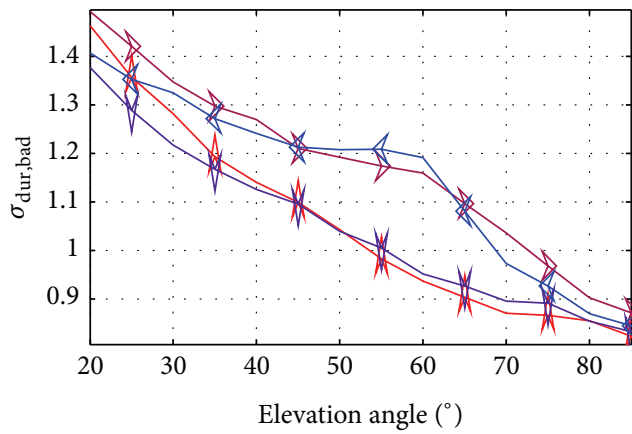

(c) Urban environment

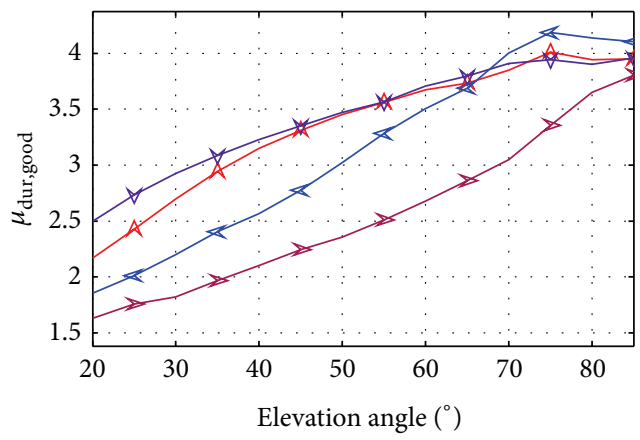

(e) Urban environment

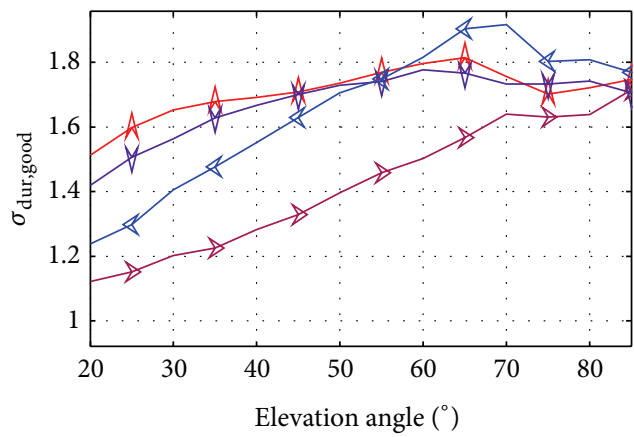

$$
\begin{aligned}
& \underset{A}{A} \text { Front } \quad \forall \text { Back } \\
& \rightarrow \text { Right } \varangle \text { Left }
\end{aligned}
$$

(g) Urban environment

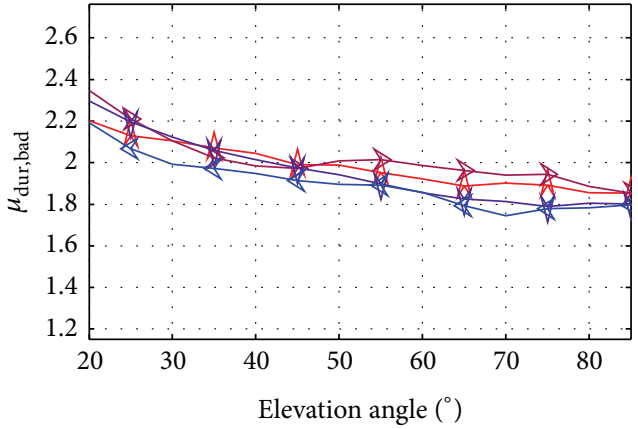

(b) Suburban environment

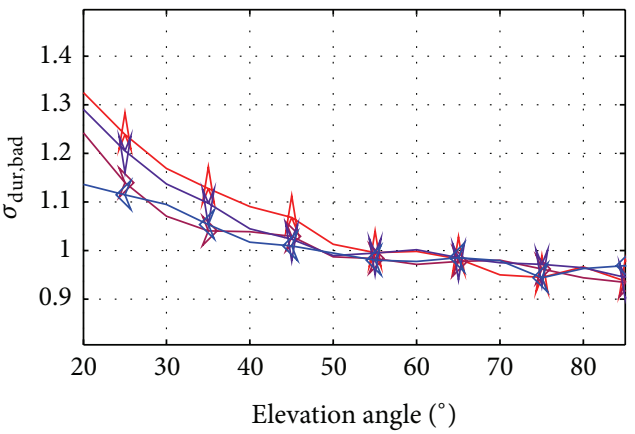

(d) Suburban environment

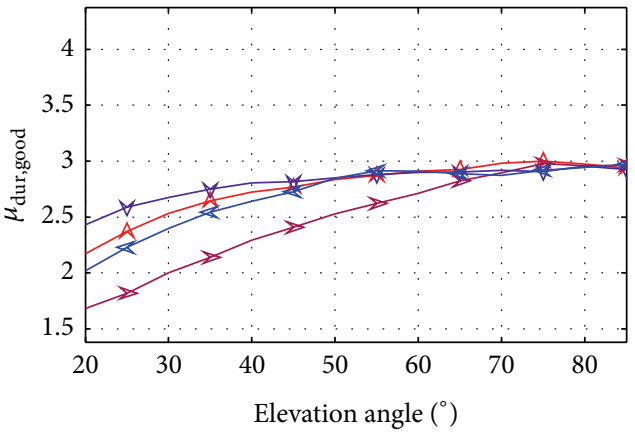

(f) Suburban environment

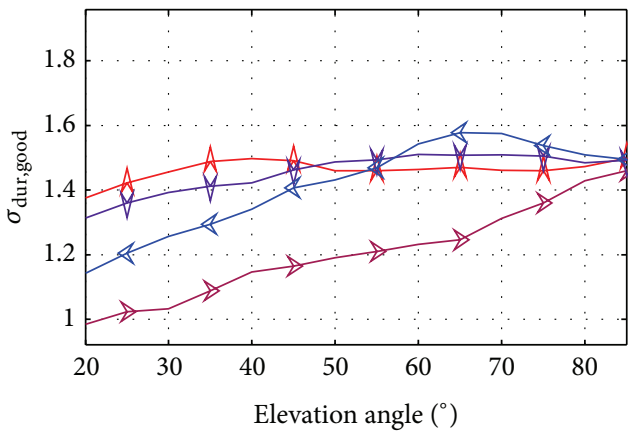

$$
A \text { Front } \forall \text { Back }
$$

(h) Suburban environment

Figure 13: The log-normal mean $\left(\mu_{\text {dur,bad }}, \mu_{\text {dur,good }}\right)$ and the log-normal standard deviation $\left(\sigma_{\text {dur,bad }}, \sigma_{\text {dur,good }}\right)$ for bad and good states, respectively. The results refer to urban (left) and suburban (right) environment for different driving directions. 


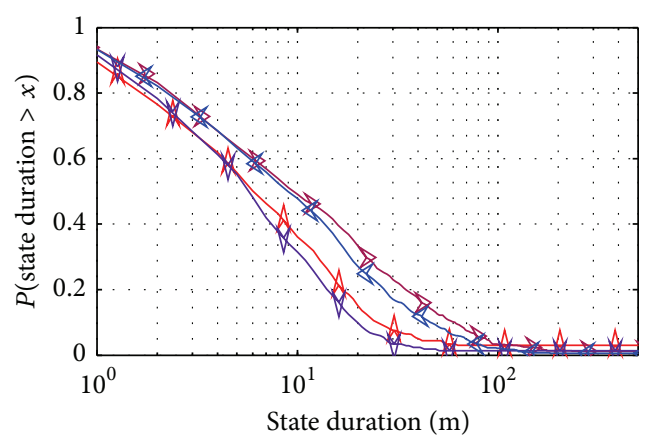

(a) Satellite at $20^{\circ}$ elevation angle in urban environment

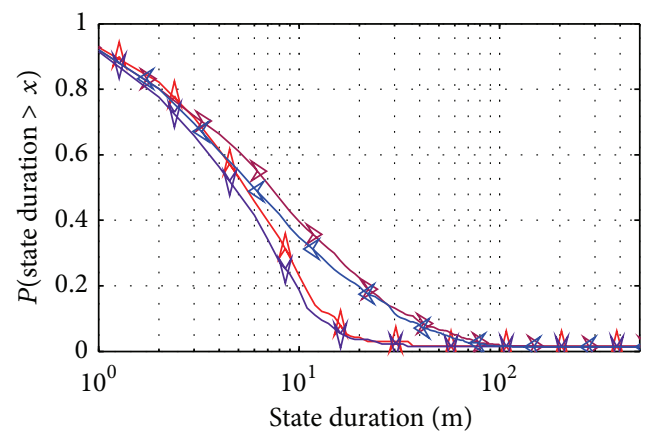

(c) Satellite at $40^{\circ}$ elevation angle in urban environment

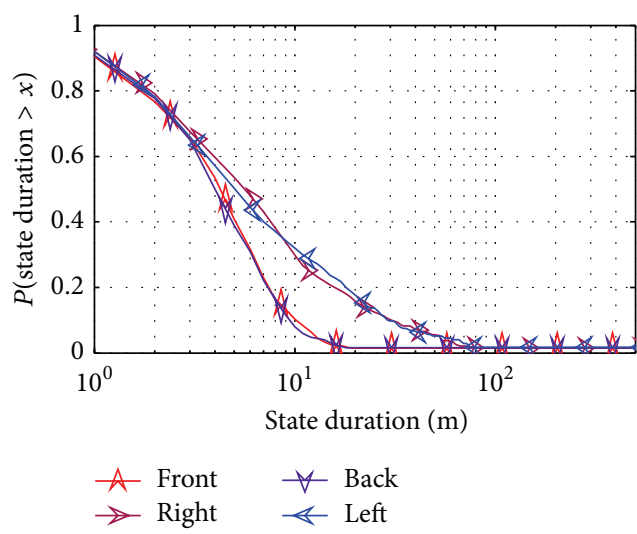

(e) Satellite at $60^{\circ}$ elevation angle in urban environment

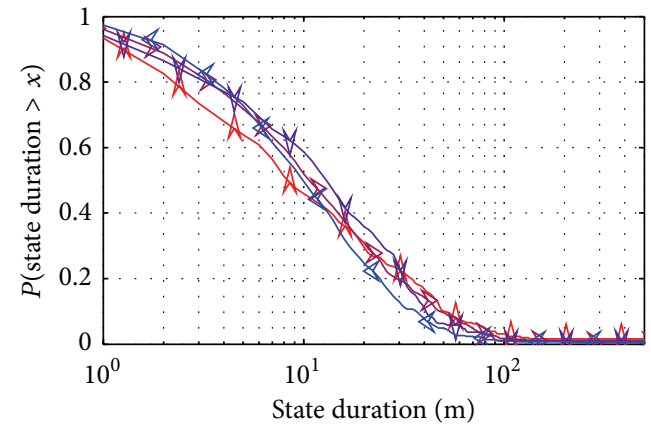

(b) Satellite at $20^{\circ}$ elevation angle in suburban environment

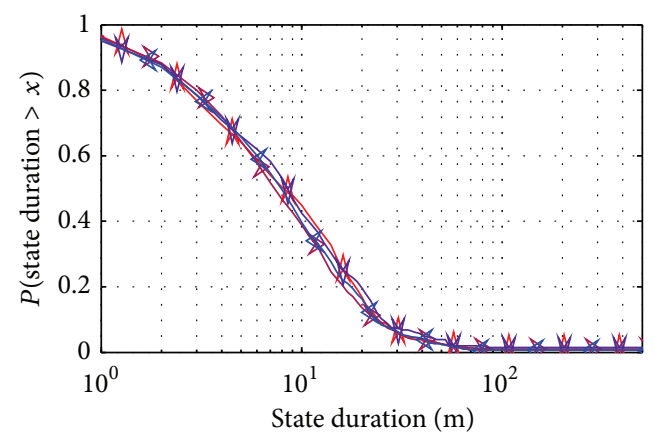

(d) Satellite at $40^{\circ}$ elevation angle in suburban environment

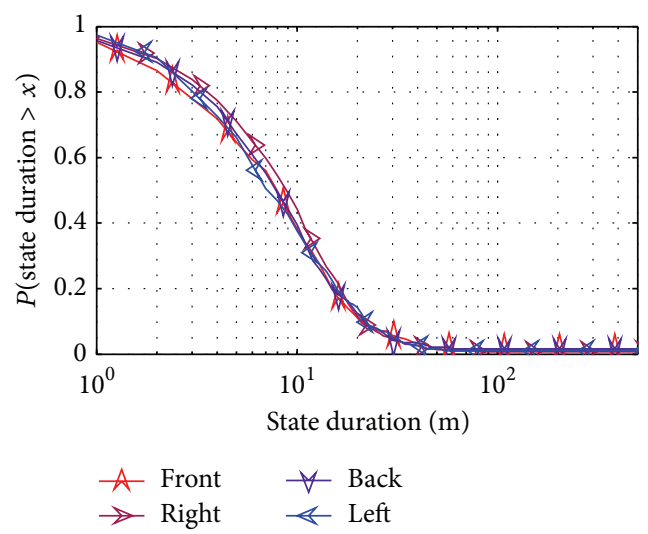

(f) Satellite at $60^{\circ}$ elevation angle in suburban environment

FIGURE 14: State duration statistics for the bad state with respect to different driving directions and elevation angles in urban (left) and suburban environment (right).

becomes visible as approaching higher elevations. In contrast to urban environment, the bad state probability for suburban does not become zero due to the trees close to the road, reaching into regions of high elevation.

The hemispheric images offer the possibility to analyze with a resolution of one degree in azimuth and in elevation. Based on the results of Figure 11 we further focus on eight exemplary satellite positions relative to the street orientation, see Figure 11. Turning clockwise, the positions are front, front-right, right, back-right, back, back-left, left, and frontleft and correspond to the azimuthal angles $0^{\circ}, 45^{\circ}, 90^{\circ}, 135^{\circ}$, $180^{\circ}, 225^{\circ}, 270^{\circ}$, and $315^{\circ}$ relative to the driving direction, respectively.

In order to model the state sequence by a Markov process, the state probability $p_{b}$, the log-normal mean of the state duration $\mu_{\text {dur }}$, and the log-normal standard deviation $\sigma_{\text {dur }}$ are of prime interest. In Figures 12 and 13 the three parameters are plotted versus the elevation angle (left for urban and on the right for suburban environment). For the sake of clarity we only show four different main positions of the satellite relative to the street orientation (front, right, back, and left). In Tables 2 and 3 , the log-normal state duration parameters $\left(\mu_{\mathrm{dur}}\right.$, 


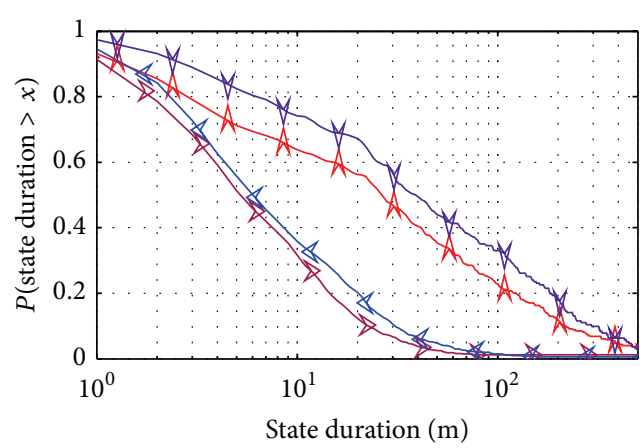

(a) Satellite at $20^{\circ}$ elevation angle in urban environment

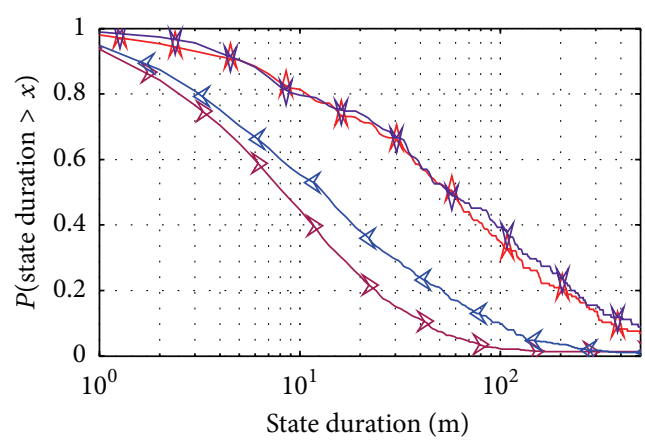

(c) Satellite at $40^{\circ}$ elevation angle in urban environment

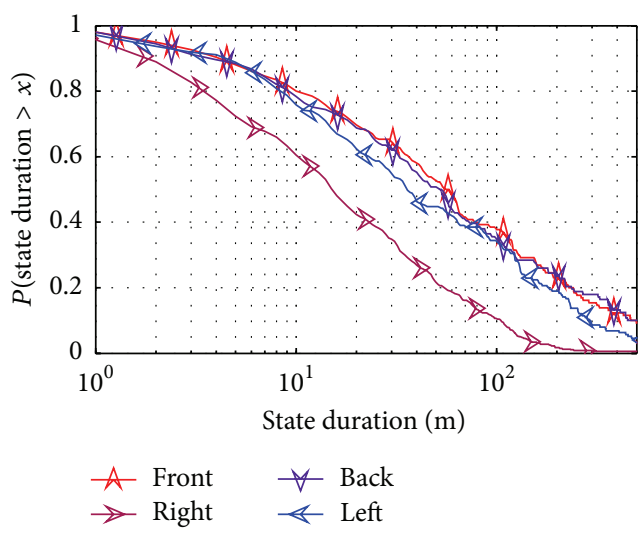

(e) Satellite at $60^{\circ}$ elevation angle in urban environment

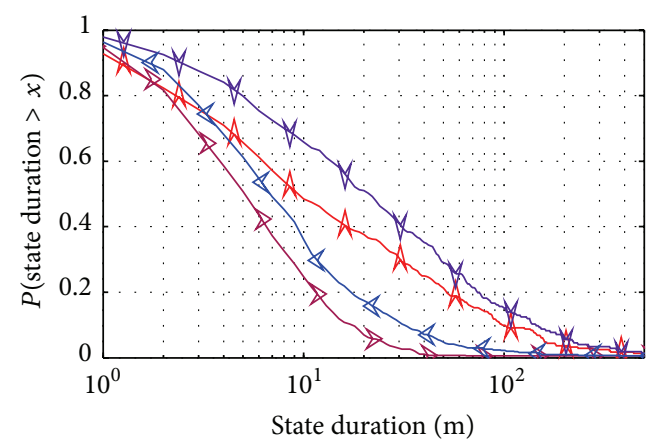

(b) Satellite at $20^{\circ}$ elevation angle in suburban environment

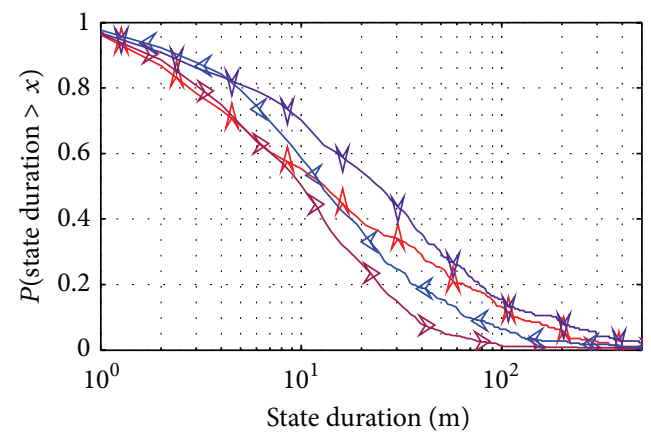

(d) Satellite at $40^{\circ}$ elevation angle in suburban environment

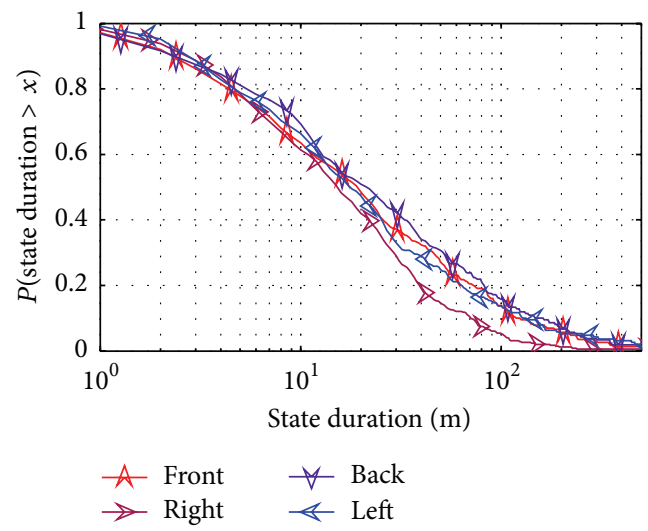

(f) Satellite at $60^{\circ}$ elevation angle in suburban environment

FIGURE 15: State duration statistics for the good state with respect to different driving directions and elevation angles in urban (left) and suburban environment (right).

$\sigma_{\text {dur }}$ ) for the urban and suburban environment are provided for eight satellite positions in azimuth and elevation angles ranging from $15^{\circ}$ to $85^{\circ}$. Due to the limited building height, the values of the bad state probability, the log-normal mean for $b a d$, and the log-normal standard deviation for bad state decrease with an increasing elevation angle. The log-normal mean good state duration and the good state log-normal standard deviation show an inverse relationship. In particular, for urban scenarios a strong difference between the values of all the parameters for different satellite positions can be noticed; for example, a difference up to $20 \%$ in service unavailability can be seen. The estimated log-normal mean $\mu_{\text {dur }}$ and the log-normal standard deviation $\sigma_{\text {dur }}$ are crucial parameters to describe the state duration probability density function (SDPDF) of the utilized semi-Markov chain (see Section 4). As we have shown that both parameters are dependent on the driving direction, we present the resulting complementary SDPDFs for the good and bad state in Figures 14 and 15, respectively. Each figure comprises urban (left) and suburban environment (right). From top to bottom, the elevation angle of the satellite is increasing from $20^{\circ}$ to $40^{\circ}$ and $60^{\circ}$, and again the four different positions in azimuth are analyzed. In urban 


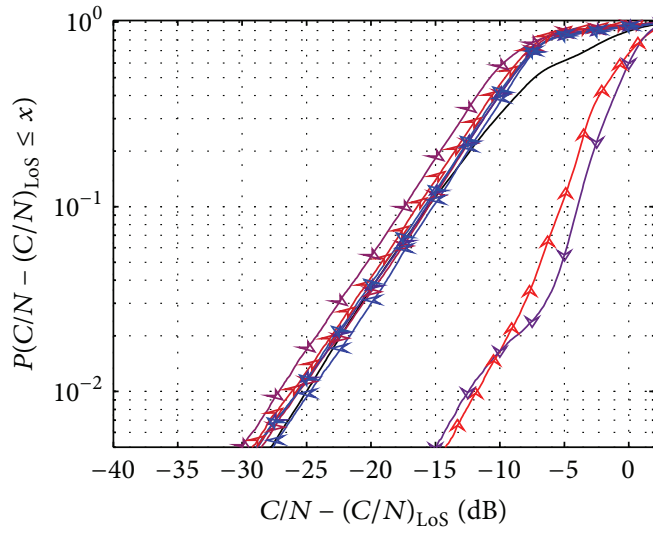

(a) Satellite at $20^{\circ}$ elevation angle

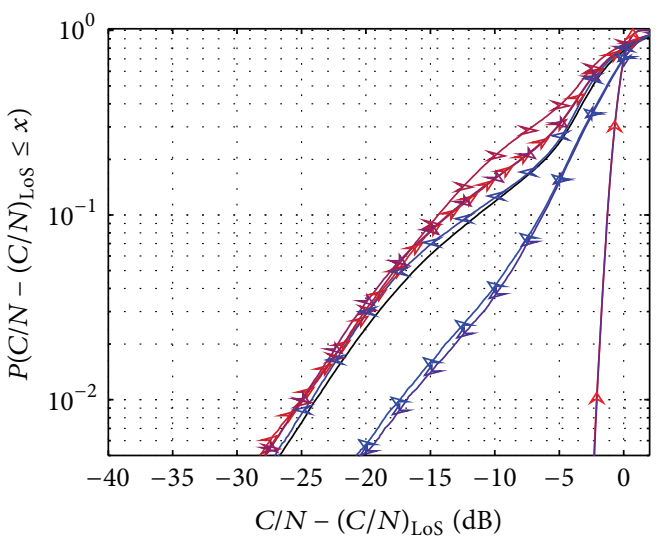

(c) Satellite at $40^{\circ}$ elevation angle

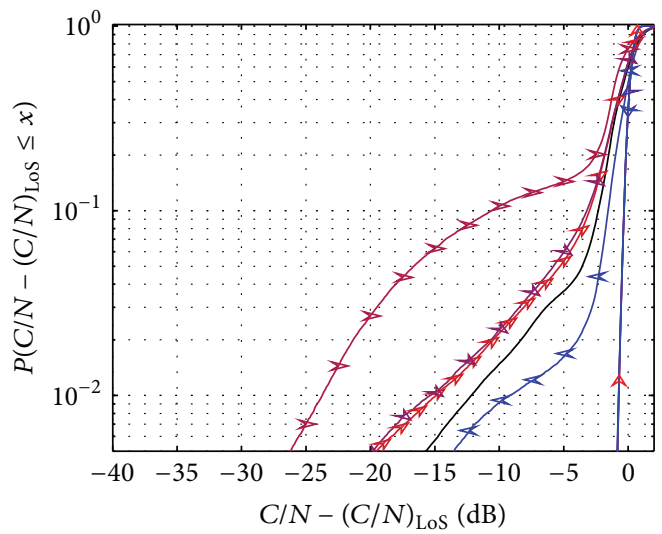

(e) Satellite at $60^{\circ}$ elevation angle

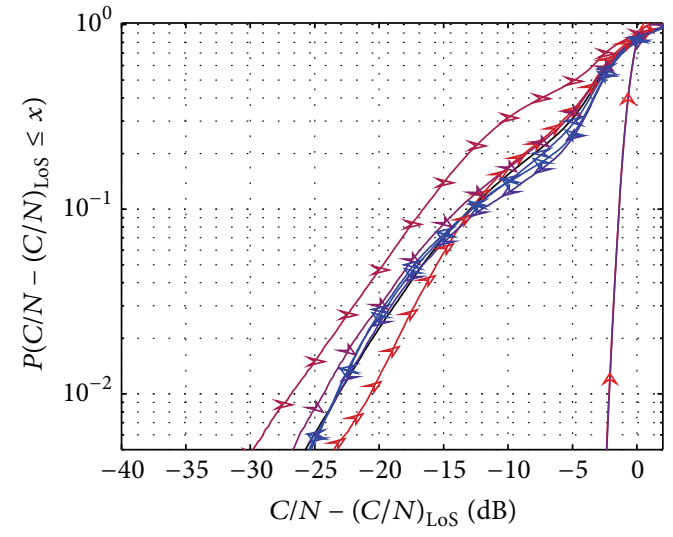

(b) Satellite at $30^{\circ}$ elevation angle

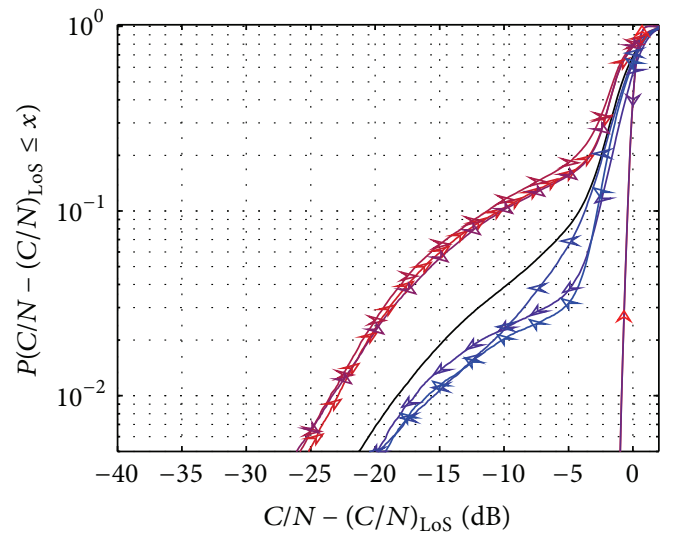

(d) Satellite at $50^{\circ}$ elevation angle

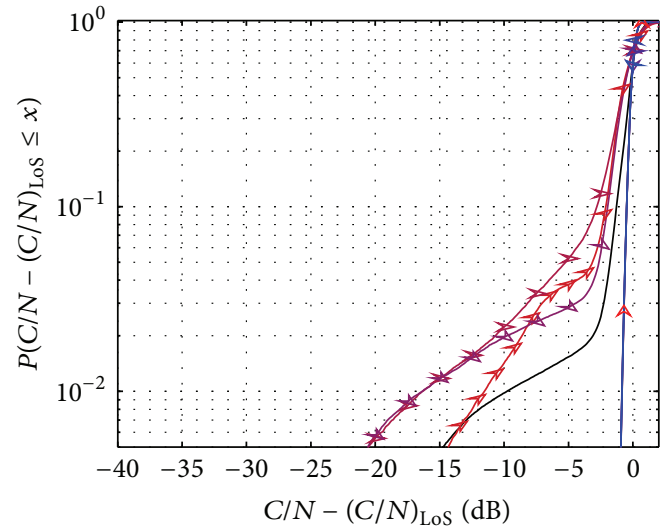

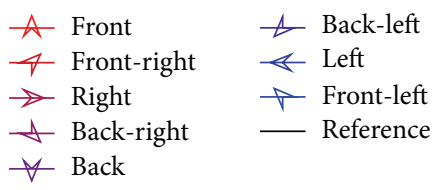

(f) Satellite at $70^{\circ}$ elevation angle

FIGURE 16: Comparison between the traditional and the proposed channel model with respect to the CDF of the received satellite signal envelope. For the results we consider data that has been collected in urban environment and each plot is related to a different elevation angle. 


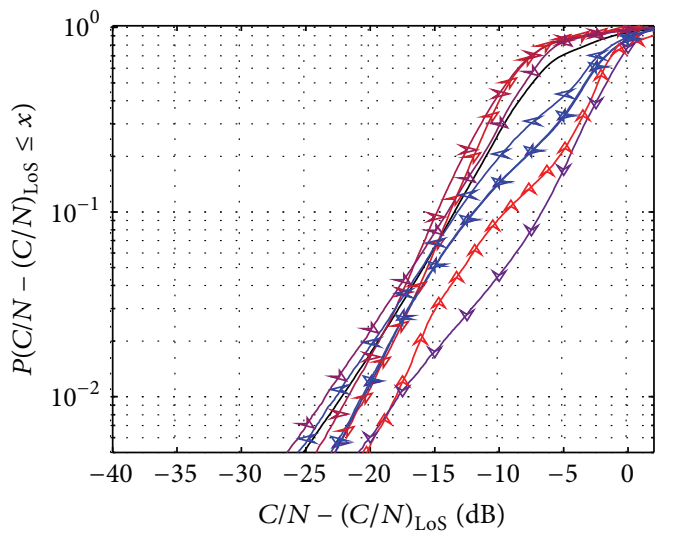

(a) Satellite at $20^{\circ}$ elevation angle

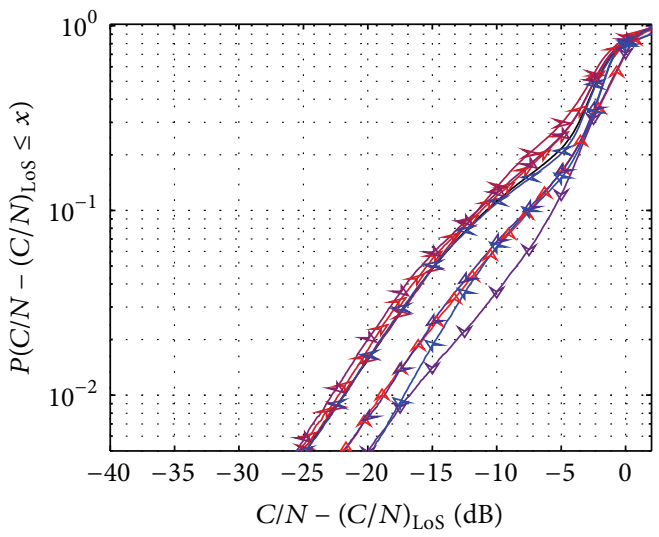

(c) Satellite at $40^{\circ}$ elevation angle

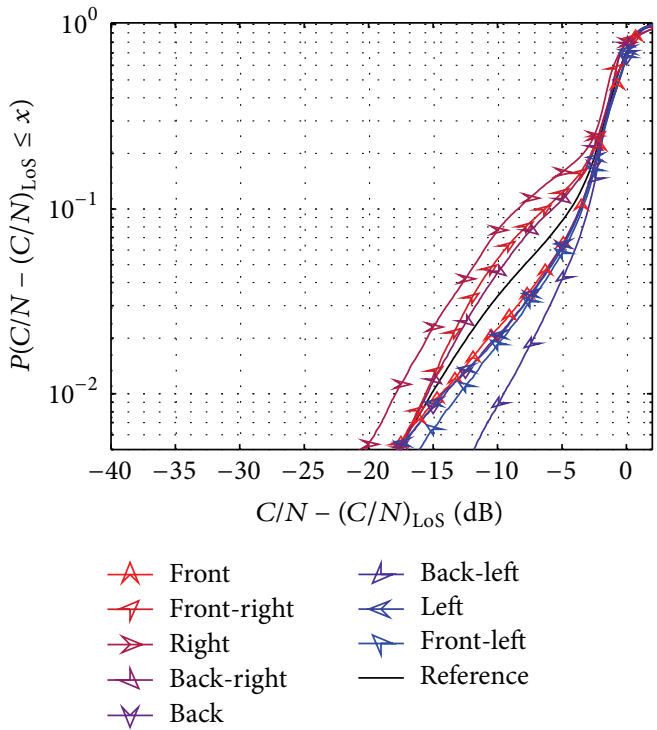

(e) Satellite at $60^{\circ}$ elevation angle

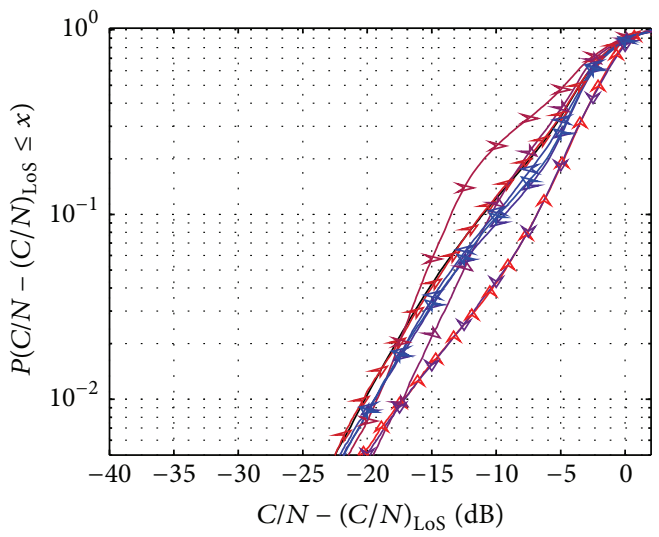

(b) Satellite at $30^{\circ}$ elevation angle

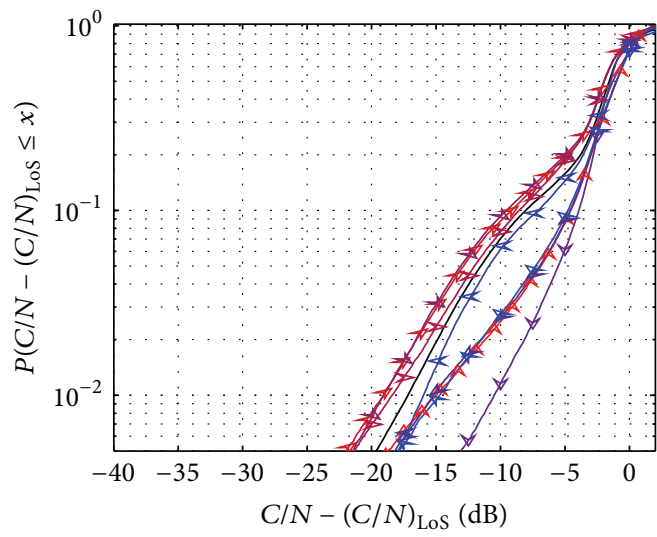

(d) Satellite at $50^{\circ}$ elevation angle
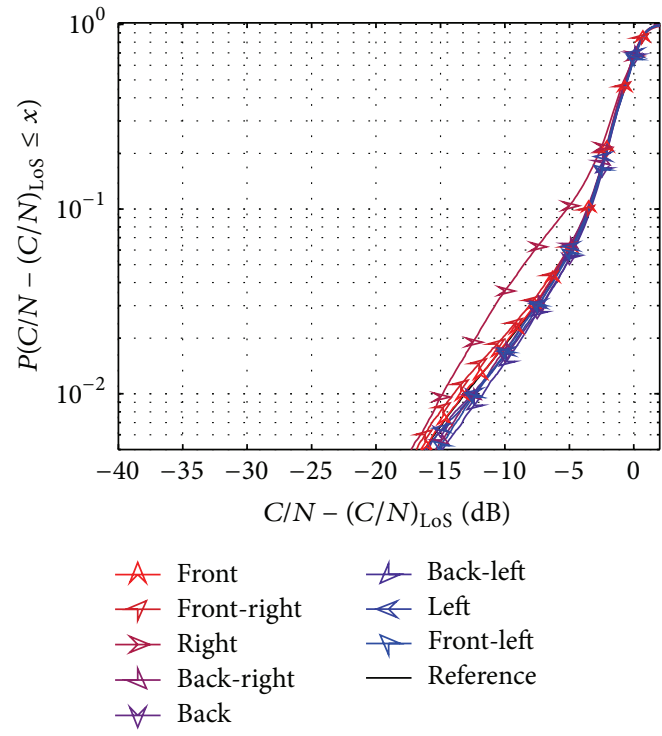

(f) Satellite at $70^{\circ}$ elevation angle

FIGURE 17: Comparison between the traditional and the proposed channel model with respect to the CDF of the received satellite signal envelope. For the results we consider data that has been collected in suburban environment and each plot is related to a different elevation angle. 
environment considerable differences in the state duration statistics can be identified, where right represents the worst case and front/back the best case. For suburban scenarios the differences in the statistical behavior decrease. In the first part of this section we reveal significant differences of the state parameters depending on the environment, elevation, and on the azimuth relative to the driving direction.

Building on the results presented so far, we now investigate the impact of the driving direction by comparing the performance of the image-based LMS model output (see Section 4). The evaluation is performed in terms of first order statistics (Cumulative Distribution Functions (CDFs)) of the LoS-normalized carrier-to-noise ratio $\left(C / N-(C / N)_{\text {LoS }}\right.$ in $\mathrm{dB}$ ), for eight exemplary satellite positions relative to the street orientation, see Figure 11. Turning clockwise, the positions are front, front-right, right, back-right, back, backleft, left, and front-left and correspond to the azimuthal angles $0^{\circ}, 45^{\circ}, 90^{\circ}, 135^{\circ}, 180^{\circ}, 225^{\circ}, 270^{\circ}$, and $315^{\circ}$ relative to the driving direction, respectively. By extracting the satellite reception states from the images for the particular satellite position (see Section 3.1), the driving direction dependency is considered within the state parameter set. The Loo parameters $\left(M_{A}, \Sigma_{A}, M P\right)$, chosen from statistical distributions, depend only on the environment and the elevation angle [5]. The results, corresponding to routes in the urban and suburban environment of Portland (Maine), are depicted in Figures 16 and 17, respectively. Each plot refers to a specific elevation angle (from $20^{\circ}$ to $70^{\circ}$ ) and the colored curves with different markers represent the eight chosen satellite positions with respect to the driving direction. As a reference, the black curve is generated by considering a route in the corresponding environment, while the heading of the vehicle is assumed to be uniformly distributed. This method represents traditional modeling without an explicit consideration of the azimuth angle.

For all plots a clear tendency of an increasing LoSnormalized $C / N$ related to an increasing elevation angle can be seen. The limited height of obstacles in the vicinity of the vehicle leads to better LoS conditions for higher elevations. At low elevation angles of the satellite $\left(20^{\circ}-30^{\circ}\right)$ the impact of the street canyon becomes obvious (Figures 16(a) and 16(b)). A satellite oriented in the driving direction (front) or in the opposite (back) leads to an almost $17 \mathrm{~dB}$ higher LoSnormalized $C / N$ compared to satellites positioned behind the buildings on the left or right side. The reference curve (ignoring the heading) is very close to the worst case.

In Figures 16(c) and 16(d), a third group with approximately $6 \mathrm{~dB}$ better than the worst case can be identified, consisting of the satellite positions front-left, back-left, and left, respectively. Here, the effect of the right-hand driving of the vehicle becomes evident; objects at the right side are closer and in relation to the position of the car, higher in elevation. The reference estimates the channel close to the worst case or close to the CDFs of the left positions. Consequently, modeling the satellite signal without any information of the heading of the vehicle leads to an overly optimistic estimation for satellites positioned at the right side.
Figure 16(e) shows the diversity of the satellite channel at an elevation of $60^{\circ}$. The CDFs of front-left and back-left superpose with front and back. The satellite at the left position behaves slightly worse. The LoS-normalized $C / N$ decreases even more for front-left and back-left $(\approx 7 \mathrm{~dB})$ and right is still the worst case. The reference CDF is approximately located in the middle of the set of curves.

For $70^{\circ}$ elevation (Figure 16(f)) the positions on the right side represent the worst case with a difference of $\approx 15 \mathrm{~dB}$ to the other superposed curves. Again, the reference curve represents almost an average of worst case and best case.

In Figure 17 the results for suburban environment are shown. Not surprisingly, the set of CDFs is closer to each other $(\leq 10 \mathrm{~dB})$; nonetheless the same worst and best cases can be identified.

\section{Conclusions}

In this contribution, we present a statistical Land Mobile Satellite (LMS) channel model, which includes the azimuth angle of the satellite with respect to the driving direction. To derive the necessary LMS model parameters for different headings and arbitrary satellite positions, an imagebased parameter estimation method is used. This allows us to reduce the measurement effort significantly, compared to a conventional RF measurement campaign. We verify the image-based method by comparing the statistical state parameters derived from images with the ones derived from the corresponding measured RF signal levels, serving as a ground truth. The results show a good match, demonstrating that the image-based approach is a reliable method for detecting the very slow fading of the received satellite signal.

The proposed LMS channel model comprises a semiMarkov model using image-based state duration parameters to generate realistic state sequences for different driving directions. In particular, the extracted state duration statistics are strongly influenced by the driving direction. The model is able to generate time-series of the received satellite signal for different azimuth positions relative to the heading. We show that varying headings lead to significant differences in the first order statistics of the carrier-to-noise ratio, demonstrating the considerable nonstationarity of the received satellite signal. Although the proposed approach provides an accurate and realistic model, only a few user input parameters are necessary. This makes it an attractive tool for designing and testing of satellite systems.

\section{Conflict of Interests}

The authors declare that there is no conflict of interests regarding the publication of this paper.

\section{Acknowledgment}

The measurements were carried out in the context of the project MiLADY (Mobile satellite channeL with Angle DiversitY), funded by the European Space Agency (ESA) under contract no. C21150. 


\section{References}

[1] F. Pérez-Fontán, M. Vázquez-Castro, C. E. Cabado, J. P. García, and E. Kubista, "Statistical modeling of the LMS channel," IEEE Transactions on Vehicular Technology, vol. 50, no. 6, pp. 15491567, 2001.

[2] R. Prieto-Cerdeira, F. Pérez-Fontán, P. Burzigotti, A. BoleaAlamaac, and I. Sanchez-Lago, "Versatile two-state land mobile satellite channel model with first application to DVB-SH analysis," International Journal of Satellite Communications and Networking, vol. 28, no. 5-6, pp. 291-315, 2010.

[3] E. Lutz, "A Markov model for correlated land mobile satellite channels," International Journal of Satellite Communications, vol. 14, no. 4, pp. 333-339, 1996.

[4] D. Arndt, T. Heyn, A. Heuberger, R. Prieto-Cerdeira, and E. Eberlein, "State modeling of the land mobile satellite channel with angle diversity," in Proceeding of the 6th European Conference on Antennas and Propagation (EuCAP '12), pp. 3140-3144, Prague, Czech Republic, March 2012.

[5] D. Arndt, T. Heyn, J. König et al., "Extended two-state narrowband LMS propagation model for S-Band," in Proceedings of the IEEE International Symposium on Broadband Multimedia Systems and Broadcasting (BMSB '12), pp. 1-6, Seoul, Republic of Korea, June 2012.

[6] D. Arndt, A. Ihlow, T. Heyn, A. Heuberger, R. Prieto-Cerdeira, and E. Eberlein, "State modelling of the land mobile propagation channel for dual-satellite systems," EURASIP Journal on Wireless Communications and Networking, vol. 2012, article 228, 2012.

[7] D. Arndt, A. Ihlow, A. Heuberger, and E. Eberlein, "State modelling of the land mobile propagation channel with multiple satellites," International Journal of Antennas and Propagation, vol. 2012, Article ID 625374, 15 pages, 2012.

[8] C. Tzaras, S. R. Saunders, and B. G. Evans, "A physical-statistical pro pagation model for diversity in mobile satellite PCN," in Proceedings of the 48th IEEE Vehicular Technology Conference (VTC '98), vol. 1, pp. 525-529, Ottawa, Canada, May 1998.

[9] C. Oestges, S. R. Saunders, and D. Vanhoenacker-Janvier, "Physical statistical modelling of the land mobile satellite channel based on ray tracing," IEE Proceedings: Microwaves, Antennas and Propagation, vol. 146, no. 1, pp. 45-49, 1999.

[10] C. Oestges and D. Vanhoenacker-Janvier, "A physical-statistical shadowing correlation model and its application to low-earthorbit systems," IEEE Transactions on Vehicular Technology, vol. 50, no. 2, pp. 416-421, 2001.

[11] C. Oestges and D. Vanhoenacker-Janvier, "Time series-based prediction of fade dynamics and shado wing correlation statistics for navigation systems," in Proceedings of the 2nd European Conference on Antennas and Propagation (EuCAP '07), pp. 1-6, IET, Edinburgh, UK, 2007.

[12] M. A. Vázquez-Castro, F. Pérez-Fontán, H. Iglesias-Salgueiro, and M. A. Barcia-Fernandez, "A simple three-segment model for shadowing cross correlation in multisatellite systems in street canyons," Microwave and Optical Technology Letters, vol. 28, no. 3, pp. 160-164, 2001.

[13] M. A. Vázquez-Castro, F. Pérez-Fontán, and S. R. Saunders, "Shadowing correlation assessment and modeling for satellite diversity in urban environments," International Journal of Satellite Communications, vol. 20, no. 2, pp. 151-166, 2002.

[14] F. Pérez-Fontán, M. A. Vázquez-Castro, C. Enjamio, and J. Pita, "Modeling the building blockage cross-correlation in multi-satellite systems," in Proceedings of the 55th Vehicular Technology Conference (VTC '02), vol. 2, pp. 928-932, May 2002.

[15] F. Pérez-Fontán, M. A. Vázquez-Castro, C. Enjamio-Cabado, and J. Pita, "Simplified multi-satellite system availability calculations using street masking functions," in Proceedings of the 55th IEEE Vehicular Technology Conference (VTC '02), vol. 4, pp. 1864-1868, May 2002.

[16] F. Pérez-Fontán, M. A. Vázquez-Castro, C. Enjamio, P. Mariño, and J. Pita, "Approximate urban area call drop-out probability estimation in mobile multi-satellite systems," International Journal of Satellite Communications and Networking, vol. 21, no. 2, pp. 183-197, 2003.

[17] F. Pérez-Fontán, S. Martínez, B. Sanmartin, C. Enjamio, P. Mariño, and F. Machado, "An enhanced Markov chain based model for the narrowband LMS channel in built-up areas," International Journal of Satellite Communications and Networking, vol. 23, no. 2, pp. 111-128, 2005.

[18] C. Meenan, M. Parks, R. Tafazolli, and B. Evans, "Availability of 1st generation satellite personal communication network service in urban environments," in Proceedings of the IEEE 48th Vehicular Technology Conference (VTC Spring '98), vol. 2, pp. 1471-1475, IEEE, Ottawa, Canada, May 1998.

[19] R. Akturan and W. J. Vogel, "Optically derived elevation angle dep endence of fading for satellite PCS," Proceedings of the 19th NASA Propagation Experimenters Meeting (NAPEX '95), vol. 1, pp. 127-132, 1995.

[20] R. Akturan and W. J. Vogel, "Path diversity for LEO satellite-PCS in the urban environment," IEEE Transactions on Antennas and Propagation, vol. 45, no. 7, pp. 1107-1116, 1997.

[21] R. Akturan, H.-P. Lin, and W. J. Vogel, "Propagation modeling in land mobile satellite systems using photogrammetry," in Proceedings of the IEEE 46th Vehicular Technology Conference, pp. 1018-1022, Atlanta, Ga, USA, May 1996.

[22] M. Rieche, D. Arndt, A. Ihlow, F. Pérez-Fontán, and G. Del Daldo, "Impact of driving direction on land mobile satellite channel modeling," in Proceedings of the 8th European Conference on Antennas and Propagation (EuCAP '14), The Hague, The Netherlands, 2014.

[23] R. Akturan and W. J. Vogel, "Photogrammetric mobile satellite service prediction," Electronics Letters, vol. 31, no. 3, pp. 165-166, 1995.

[24] L. Castanet, "Influence of the variability of the propagation channel on mobile, fixed multimedia and optical satellite communications," in Mobile/Indoor Multipath, L. Castanet, Ed., Shaker, Aachen, Germany, 2008.

[25] A. G. Kanatas and P. Constantinou, "A narrowband land mobile satellite channel software simulator for urban environments," International Journal of Satellite Communications, vol. 18, no. 1, pp. 17-45, 2000.

[26] M. Döttling, A. Jahn, D. Didascalou, and W. Wiesbeck, “Twoand three-dimensional ray tracing applied to the land mobile satellite (LMS) propagation channel," IEEE Antennas and Propagation Magazine, vol. 43, no. 6, pp. 27-37, 2001.

[27] C. Loo, "A statistical model for a land mobile satellite link," IEEE Transactions on Vehicular Technology, vol. 34, no. 3, pp. 122-127, 1985.

[28] G. E. Corazza and F. Vatalaro, "A statistical model for land mobile satellite channels and its application to nongeostationary orbit systems," IEEE Transactions on Vehicular Technology, vol. 43, no. 3, pp. 738-742, 1994. 
[29] H. Suzuki, "A statistical model for urban radio propogation," IEEE Transactions on Communications, vol. 25, no. 7, pp. 673680, 1977.

[30] E. Lutz, D. Cygan, M. Dippold, F. Dolainsky, and W. Papke, "The land mobile satellite communication channel: recording, statistics, and channel model," IEEE Transactions on Vehicular Technology, vol. 40, no. 2, pp. 375-386, 1991.

[31] Y. Karasawa, K. Minamisono, and T. Matsudo, "A propaga tion channel model for personal mobilesatellite services," in Proceedings of Progress of Electromagnetic Research Symposium of the European Space Agency (ESA '95), pp. 11-15, Noordwijk, The Netherlands, July 1995.

[32] S. Scalise, C. Alasseur, L. Husson, and H. Ernst, "Accurate and novel modeling of the land mobile satellite channel using reversible jump markov chain monte carlo technique," in Proceedings of the IEEE Global Telecommunications Conference (GLOBECOM '06), pp. 1-6, San Francisco, Calif, USA, November 2006.

[33] M. Milojević, M. Haardt, A. Heuberger, and E. Eberlein, "Channel state modeling for single and multiple satellite broadcasting systems," in Proceedings of the International Workshop on Satellite and Space Communications (IWSSC '08), pp. 102106, Toulouse, France, October 2008.

[34] H.-P. Lin, R. Akturan, and W. J. Vogel, "Satellite-PCS channel simulation in mobile user environments using photogrammetry and Markov chains," Wireless Networks, vol. 3, no. 4, pp. 299308, 1997.

[35] M. Rieche, D. Arndt, A. Ihlow, and G. Del Galdo, "State modeling of the land mobile satellite channel by an image-based approach," in Proceedings of the 7th European Conference on Antennas and Propagation (EuCAP '13), pp. 672-676, Gothenburg, Sweden, April 2013.

[36] E. Eberlein, A. Heuberger, and T. Heyn, "Channel models for systems with angle diversity-the MiLADY project," in Proceedings of the ESA Workshop on Radiowave Propagation Models, Tools and Data for Space Systems, Noordwijk, The Netherlands, December 2008.

[37] L. E. Bråten and T. Tjelta, "Semi-Markov multistate modeling of the land mobile propagation channel for geostationary satellites," IEEE Transactions on Antennas and Propagation, vol. 50, no. 12, pp. 1795-1802, 2002.

[38] J. König, D. Arndt, A. Ihlow, and A. Heuberger, "Estimation of fading parameter correlation for modeling the land mobile satellite channel," in Proceedings of the IEEE International Symposium on Broadband Multimedia Systems and Broadcasting (BMSB '12), Seoul, Republic of Korea, June 2012. 

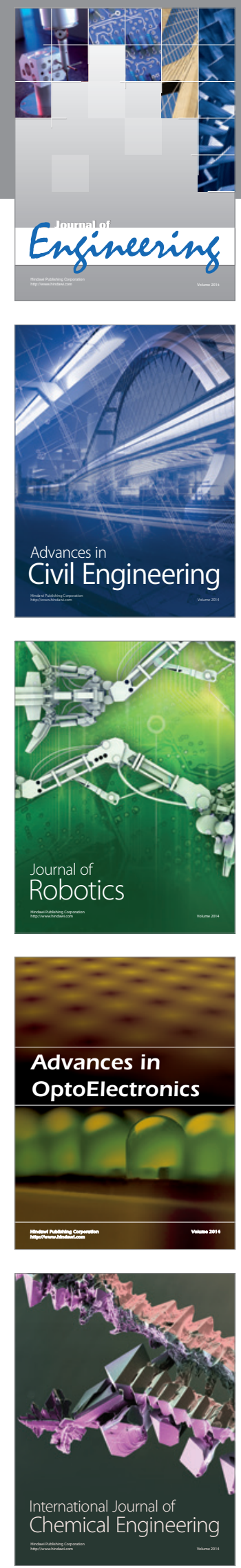

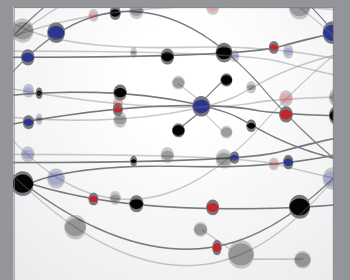

The Scientific World Journal
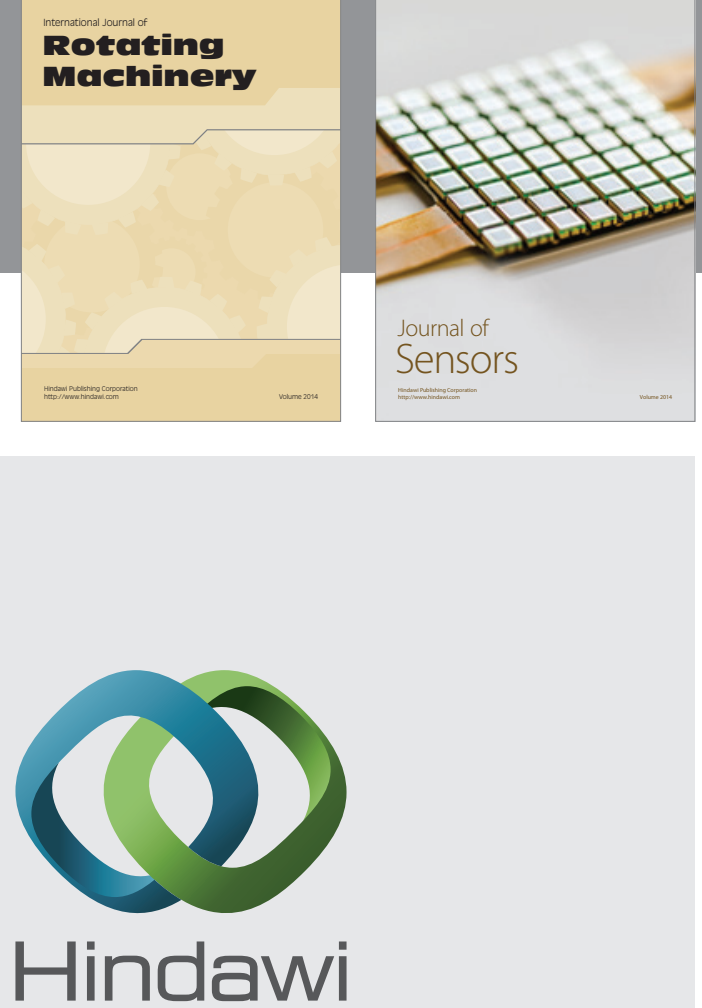

Submit your manuscripts at http://www.hindawi.com
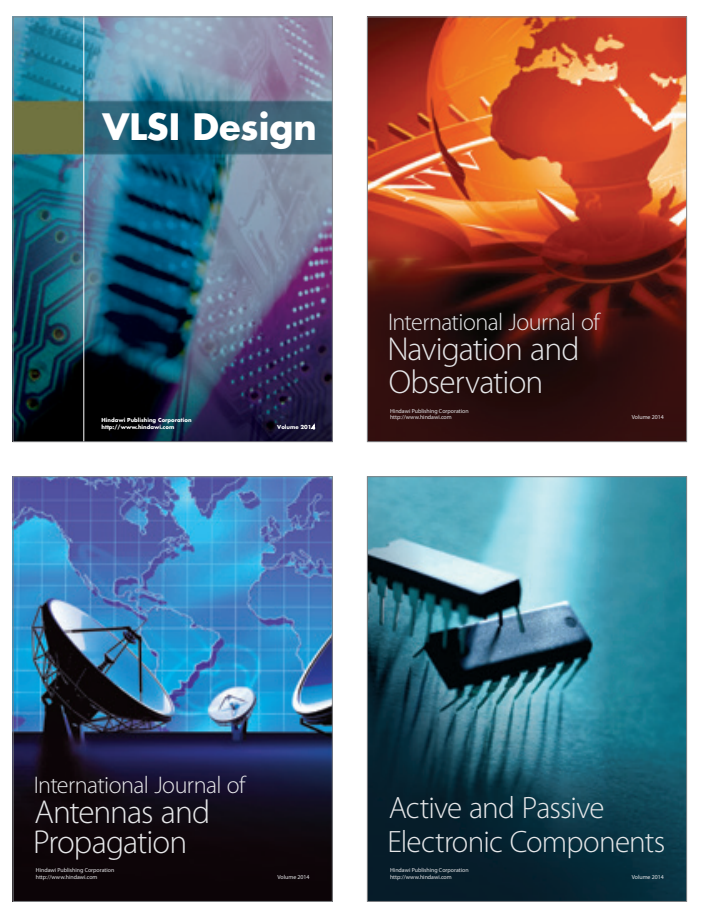
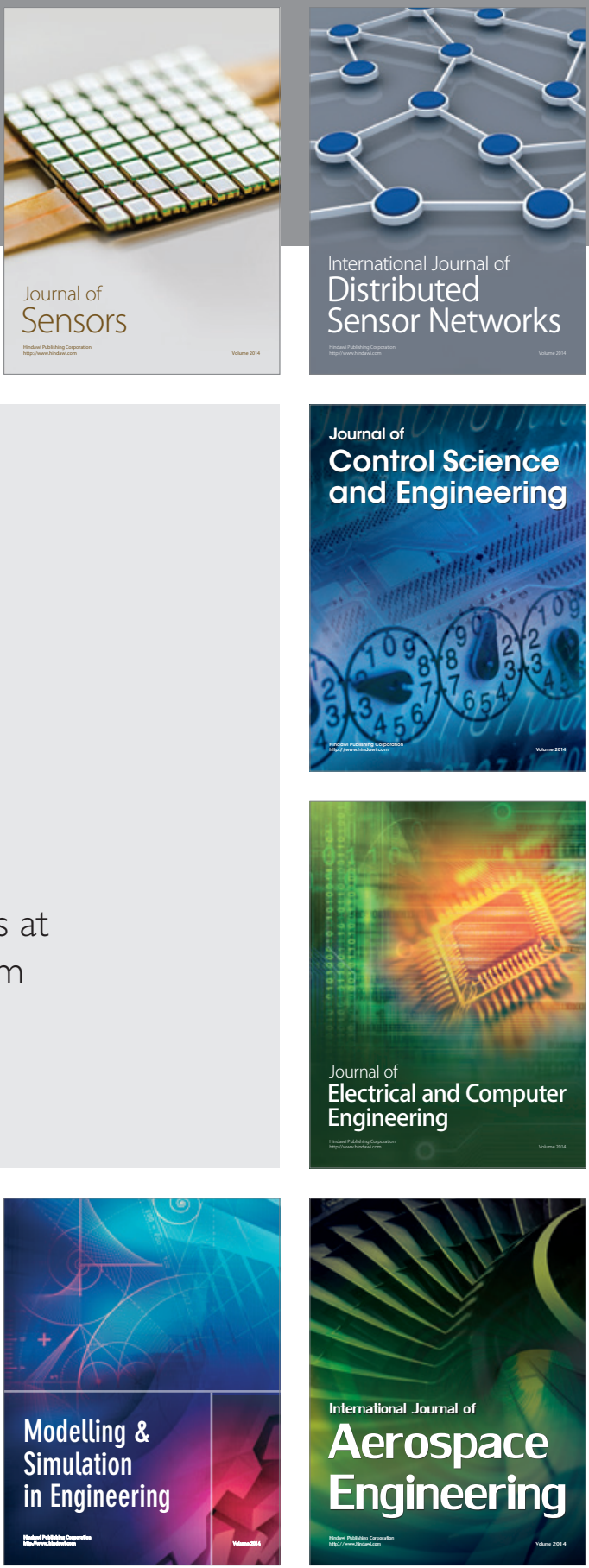

Journal of

Control Science

and Engineering
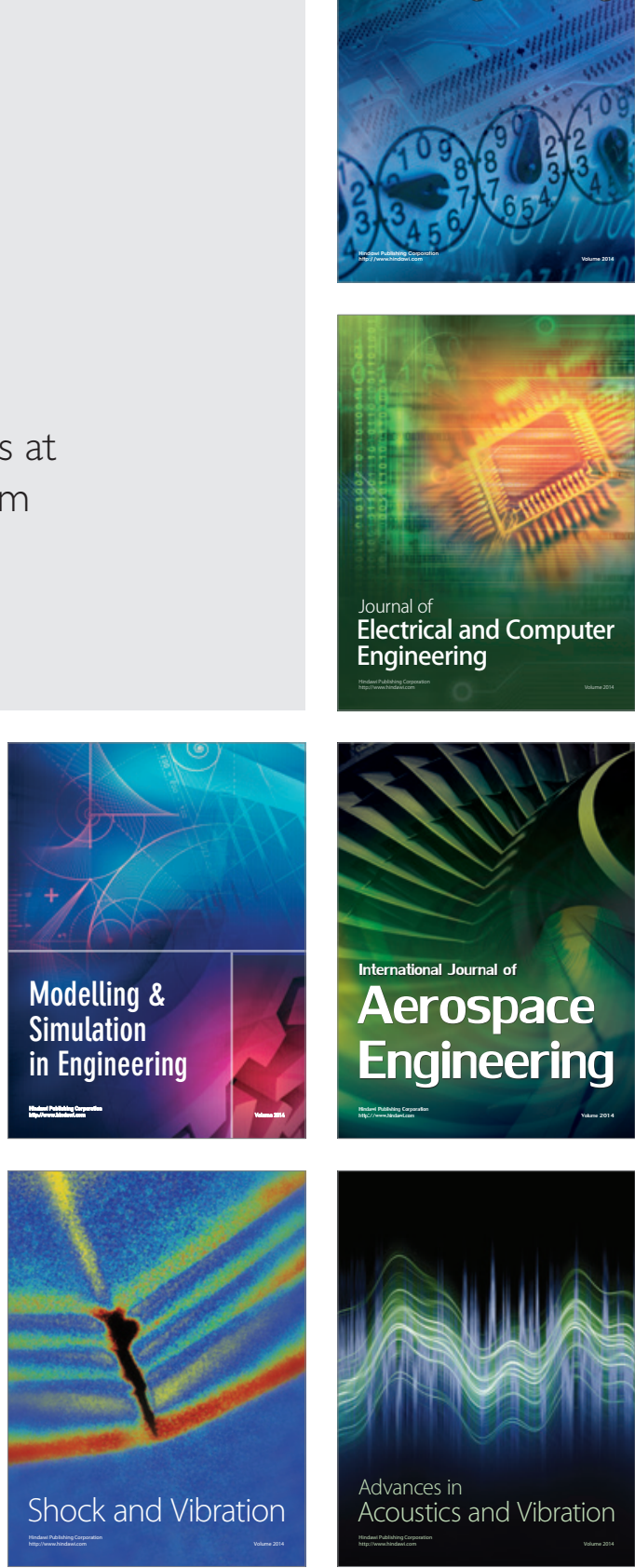